\title{
Comparison of the $\mathrm{Z} / \gamma^{*}+$ jets to $\gamma+$ jets cross sections in pp collisions at $\sqrt{s}=8 \mathrm{TeV}$
}

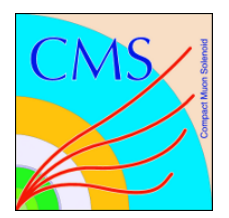

\section{The CMS collaboration}

\section{E-mail: cms-publication-committee-chair@cern.ch}

ABSTRACT: A comparison of the differential cross sections for the processes $\mathrm{Z} / \gamma^{*}+$ jets and photon $(\gamma)+$ jets is presented. The measurements are based on data collected with the CMS detector at $\sqrt{s}=8 \mathrm{TeV}$ corresponding to an integrated luminosity of $19.7 \mathrm{fb}^{-1}$. The differential cross sections and their ratios are presented as functions of $p_{\mathrm{T}}$. The measurements are also shown as functions of the jet multiplicity. Differential cross sections are obtained as functions of the ratio of the $\mathrm{Z} / \gamma^{*} p_{\mathrm{T}}$ to the sum of all jet transverse momenta and of the ratio of the $\mathrm{Z} / \gamma^{*} p_{\mathrm{T}}$ to the leading jet transverse momentum. The data are corrected for detector effects and are compared to simulations based on several QCD calculations.

Keywords: Jets, Hadron-Hadron Scattering, Beyond Standard Model, QCD, Photon production

ARXIV EPRINT: 1505.06520 


\section{Contents}

1 Introduction 1

2 CMS detector $\quad 2$

3 Monte Carlo samples 3

4 Event selection and object reconstruction 5

5 Background determination and unfolding $\quad 7$

$\begin{array}{ll}5.1 \text { The Z + jets selection } & 7\end{array}$

5.2 The $\gamma+$ jets selection 9

6 Systematic uncertainties $\quad 10$

7 Results $\quad 12$

$\begin{array}{lll}7.1 & \text { Differential cross sections } & 12\end{array}$

$\begin{array}{lll}7.2 & \text { The } \mathrm{Z} / \gamma \text { ratio } & 19\end{array}$

$\begin{array}{lll}8 & \text { Summary } & 21\end{array}$

$\begin{array}{lr}\text { The CMS collaboration } & 28\end{array}$

\section{Introduction}

The associated production of a $\mathrm{Z} / \gamma^{*}$ or a $\gamma$ with one or more jets has been extensively studied in proton-proton collisions at the CERN LHC, by both the CMS [1-5] and ATLAS [6, 7] Collaborations. Precise measurements of these processes provide important tests of the Standard Model (SM) as well as crucial inputs in the determination of parton densities in the proton [8]. Such measurements can improve the validation and tuning of the models used in Monte Carlo (MC) simulation. These processes are also important backgrounds in searches for new physics.

In the limit of high transverse momentum of the vector boson $V\left(p_{\mathrm{T}}^{V}\right)$ and at leading order (LO) in perturbative quantum chromodynamics (QCD), effects due to the mass of the $\mathrm{Z}$ boson $\left(m_{\mathrm{Z}}\right)$ are small, and the cross section ratio of $\mathrm{Z}+$ jets to $\gamma+$ jets as a function of $p_{\mathrm{T}}^{V}$ is expected to become constant, reaching a plateau for $p_{\mathrm{T}}^{V} \gtrsim 300 \mathrm{GeV}$ [8]. (In this paper, production of $\mathrm{Z} / \gamma^{*}+$ jets is denoted by $\mathrm{Z}+$ jets.) A QCD calculation at next-to-leading order (NLO) for $\mathrm{pp} \rightarrow \mathrm{Z}+$ jets and $\mathrm{pp} \rightarrow \gamma+$ jets was provided by the BLACKHAT Collaboration [9]. The NLO QCD corrections tend to lead to a decrease in the plateau value of the cross section ratio, while electroweak (EW) corrections are relatively small. However, 
at higher energies, EW corrections and QCD processes can introduce a dependence of the cross section on logarithmic terms of the form $\ln \left(p_{T}^{\mathrm{Z}} / m_{\mathrm{Z}}\right)$ that can become large and pose a challenge for perturbative calculations such as BLACKHAT+SHERPA [10]. A precise measurement of the ( $\mathrm{pp} \rightarrow \mathrm{Z}+$ jets $) /(\mathrm{pp} \rightarrow \gamma+$ jets $)$ cross section ratio provides important information about the higher-order effects of these large logarithmic corrections at higher $p_{\mathrm{T}}$.

In addition, searches for new particles involving final states characterized by the presence of large missing transverse energy $\left(\not_{\mathrm{T}}\right)$ and hard jets, as described for example in refs. [11] and [12], use the $\gamma+$ jets process to model the invisible $\mathrm{Z}$ decays, $\mathrm{Z} \rightarrow \nu \bar{\nu}$, since the $\gamma+$ jets cross section is larger than the $\mathrm{Z}+$ jets process where the $\mathrm{Z}$ decays to leptons. Measurements of the cross section ratio for $Z+$ jets and $\gamma+$ jets can help reduce uncertainties related to the $\mathrm{Z} \rightarrow \nu \bar{\nu}$ background estimation in these searches.

We present precise measurements of both production cross sections and the cross section ratio for these two processes as a function of $p_{\mathrm{T}}^{V}$. The results are compared with theoretical estimations. The data sample was collected at the LHC during the 2012 run with the CMS detector in proton-proton collisions at a center-of-mass energy $\sqrt{s}=8 \mathrm{TeV}$

and corresponds to an integrated luminosity of $19.7 \mathrm{fb}^{-1}$. The $\mathrm{Z}$ bosons are identified via their decays to pairs of electrons or muons. Measurements are made for different jet multiplicities $\left(n_{\text {jets }} \geq 1,2,3\right)$ and for a subset requiring a large hadronic transverse energy ( $H_{\mathrm{T}}>300 \mathrm{GeV}$, where $H_{\mathrm{T}}$ is the scalar sum of all selected jet $p_{\mathrm{T}}$ after jet identification). These requirements are meant to mimic the phase space requirements for analyses searching for new physics with an all-hadronic signature.

This paper is organized as follows: section 2 provides a brief description of the CMS detector; section 3 gives details of the Monte Carlo generators used in this analysis; section 4 describes the event selection; section 5 contains details about the background subtraction and the unfolding of the detector effects; section 6 discusses the sources of systematic uncertainties; section 7 presents the cross section and cross section ratio measurements for $\mathrm{Z}+$ jets and $\gamma+$ jets production. Section 8 concludes with a summary of our results.

\section{CMS detector}

The central feature of the CMS apparatus is a superconducting solenoid of $6 \mathrm{~m}$ internal diameter, providing a magnetic field of $3.8 \mathrm{~T}$. The CMS experiment uses a right-handed coordinate system, with the origin at the nominal interaction point, the $x$ axis pointing to the center of the LHC ring, the $y$ axis pointing up (perpendicular to the LHC ring), and the $z$ axis along the anticlockwise-beam direction. The polar angle $\theta$ is measured from the positive $z$ axis and the pseudorapidity is defined as $\eta=-\ln [\tan (\theta / 2)]$.

Within the superconducting solenoid volume are a silicon pixel and strip tracker, a lead tungstate crystal electromagnetic calorimeter (ECAL), and a brass and scintillator hadron calorimeter. Each subdetector is composed of a barrel and two endcap sections. Muons are measured in gas-ionization detectors embedded in the steel flux-return yoke outside the solenoid. Extensive forward calorimetry complements the coverage provided by the barrel and endcap detectors. In the barrel section of the ECAL, an energy resolution of about $1 \%$ is achieved for unconverted or late-converting photons in the tens of $\mathrm{GeV}$ energy range. 
The remaining barrel photons have a resolution of about $1.3 \%$ up to a pseudorapidity of $|\eta|=1$, rising to about $2.5 \%$ at $|\eta|=1.4$. In the endcaps, the resolution of unconverted or late-converting photons is about $2.5 \%$, while the remaining endcap photons have a resolution between 3 and 4\% [13]. The dielectron mass resolution for $\mathrm{Z} \rightarrow$ ee decays when both electrons are in the ECAL barrel is $1.8 \%$, and is $2.7 \%$ when both electrons are in the endcaps. The electron momenta are estimated by combining energy measurements in the ECAL with momentum measurements in the tracker [14]. Muons are measured in the pseudorapidity range $|\eta|<2.4$, with detection planes made using three technologies: drift tubes, cathode strip chambers, and resistive-plate chambers. Matching muons to tracks measured in the silicon tracker results in a relative transverse momentum resolution for muons with $20<p_{\mathrm{T}}<100 \mathrm{GeV}$ of $1.3-2.0 \%$ in the barrel and better than $6 \%$ in the endcap. The $p_{\mathrm{T}}$ resolution in the barrel is better than $10 \%$ for muons with $p_{\mathrm{T}}$ up to $1 \mathrm{TeV}$ [15]. A more detailed description of the CMS system can be found in ref. [16].

\section{Monte Carlo samples}

Monte Carlo simulation samples are used to correct the data for acceptance and efficiency and for the $V+$ jets processes. They are also used to estimate the background to the $\mathrm{Z}+$ jets signal.

The $\mathrm{Z}+$ jets signal is generated with the MADGRAPH (version 5.1.3.30) [17] program. The leading-order multiparton matrix element (ME) calculation includes up to four partons (gluons and quarks) in the final state. The showering and hadronization of the partons, as well as the underlying event, are modeled by PYTHIA (version 6.4.26) [18] with the Z2* tune [19]. The $k_{\mathrm{T}}$ MLM matching scheme [20] with a matching parameter of $20 \mathrm{GeV}$ is applied to avoid a double counting of final states arising in the ME calculation and the parton shower (PS). The events are generated with the CTEQ6L1 [21] parton distribution functions (PDF) and rescaled using a global next-to-next-to-leading-order (NNLO) $K$ factor to match the inclusive cross section calculated with FEWZ 3.1 [22]. Backgrounds to $\mathrm{Z}+$ jets are generated using MADGRAPH with the same configuration as the signal events. These include top quark-antiquark pairs (t⿹弋龴) and EW backgrounds, such as $\mathrm{W}+$ jets and diboson processes (WZ, ZZ, WW).

The $\mathrm{Z}+$ jets signal and $\mathrm{t} \overline{\mathrm{t}}$ background processes are also generated with SHERPA (version 1.4.2) [23], using the CT10 PDF [24]. The cross section for the signal is also rescaled using a

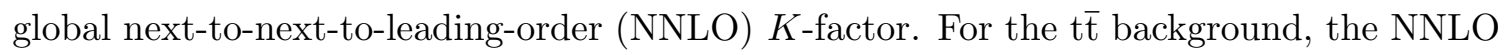
calculation provided by ref. [25] is used to calculate the NNLO $K$-factor. The background yields from EW processes are rescaled using the MCFM [26] NLO cross sections.

In addition to these general purpose MC signal data sets, we use an NLO perturbative QCD calculation of $\mathrm{Z}+$ jets from the BLACKHAT Collaboration [9], which is available for a $\mathrm{Z}$ boson accompanied by up to three jets. These simulations use MSTW2008nlo68cl [27] with $\alpha_{S}=0.119$ as the PDF set, and the renormalization and factorization scales $\left(\mu_{R}\right.$ and $\mu_{F}$, respectively) are set to

$$
\mu_{R}=\mu_{F}=H_{\mathrm{T}}^{p}+E_{\mathrm{T}}^{\mathrm{Z}} \equiv \sum_{j} p_{\mathrm{T}}^{j}+\sqrt{m_{\mathrm{Z}}^{2}+\left(p_{\mathrm{T}}^{\mathrm{Z}}\right)^{2}},
$$


where $p_{\mathrm{T}}^{j}$ is the transverse momentum of the $j$ th parton in the event and $H_{\mathrm{T}}^{p}$ is the scalar $p_{\mathrm{T}}$ sum of all outgoing partons with $p_{\mathrm{T}}>20 \mathrm{GeV}$. The CT10 and NNPDF2.3 [28] PDF sets with $\alpha_{S}=0.119$ are used as a cross check and to estimate the theoretical systematic uncertainties.

The BlackHAT+SHERPA simulated events are organized into different types of processes to facilitate the calculation. An NLO estimation at $n$ jet level is obtained by combining tree-level (LO) calculations from the $n+1$ jet case to $n$ jet tree- and loop-level calculations. The Born and real emission calculations at both $n$ and $n+1$ jet levels are supplied by SHERPA, while BLACKHAT provides the NLO virtual loop-level correction terms. (For simplicity, BLACKHAT+SHERPA will be referred to as BlackHAT.) The structure of the generated files and the preselections used in the simulation, as well as more details about BlaCKHAT, are described in ref. [10].

The $\gamma+$ jets signal is simulated by MADGRAPH, including up to four-parton final states in addition to the photon. Fixed-order cross section calculations for $\gamma+$ jets are affected by an instability due to dependencies on soft-gluon radiation, which can be overcome using allorder resummation $[29,30]$. The background contribution due to multijets is determined using control samples in data. The uncertainty in the photon purity is estimated with MC background samples simulated with PYTHIA6. Multijet events in the PYTHIA6 sample with signal-like behavior can be enhanced by applying a filter that requires jet signatures with large electromagnetic deposits in the final state, e.g., jets with hadrons decaying into high- $p_{\mathrm{T}}$ photons. As an alternative method of estimating this background, we use a MADGRAPH sample that includes jet production with as few as two and as many as four outgoing partons in the ME calculation.

We also simulate the $\gamma+$ jets signal using BLACKHAT. The overall procedure is analogous to the $\mathbf{Z}+$ jets BlACKHAT samples, and $\gamma+$ jets samples are available for $\gamma+1,2$, and 3 jets. For $\gamma+$ jets, we use the following renormalization and factorization scales:

$$
\mu_{R}=\mu_{F}=H_{\mathrm{T}}^{p}+E_{\mathrm{T}}^{\gamma} \equiv \sum_{j} p_{\mathrm{T}}^{j}+p_{\mathrm{T}}^{\gamma}
$$

The BLACKHAT production requires that the photons satisfy the Frixione cone isolation condition [31]

$$
\sum_{i} E_{\mathrm{T}}^{i} \Theta\left(\delta-R_{i \gamma}\right) \leq \mathcal{H}(\delta)
$$

for all $\delta$ less than $\delta_{0}$ around the axis of the photon. Here, $R_{i \gamma}$ is the distance in $\eta$ and azimuthal angle $\phi$ between the $i^{\text {th }}$ parton and the photon, and $\Theta$ is the step function. The function $\mathcal{H}(\delta)$ is chosen such that it vanishes as $\delta \rightarrow 0$. In particular,

$$
\mathcal{H}(\delta)=E_{\mathrm{T}}^{\gamma} \epsilon\left(\frac{1-\cos \delta}{1-\cos \delta_{0}}\right)^{n}
$$

The Frixione cone in effect only adds contributions from partons which are within $\delta_{0}$ of the photon. In the BlackHat samples, $\epsilon=0.025, \delta_{0}=0.4$, and $n=2$. These were selected because a Frixione cone with these choices mimics the selections in the true on-shell 
photon definition at particle level. Photon distributions using Frixione cone requirements are found to agree with those using cone isolation to within 1-2\% [32].

The simulation of the CMS detector is based on the Geant4 package [33]. The simulated events used for the detector level MC estimations are reconstructed following the same procedures used for the data. For our run, the average number of inelastic protonproton collisions occurring per LHC bunch crossing was 21. The correct distribution of the number of pileup events overlapping the hard interaction process per bunch crossing is taken into account in the MC by reweighting the simulated minimum bias events to match the spectrum of pileup interactions observed in data.

\section{Event selection and object reconstruction}

The selection of $\mathrm{Z}+$ jets events begins by requiring two same-flavor high- $p_{\mathrm{T}}$ leptons (electrons or muons) at trigger level. The $p_{\mathrm{T}}$ threshold of the trigger objects is $17 \mathrm{GeV}$ for the leading muon (the muon with the largest $p_{\mathrm{T}}$ ) and $8 \mathrm{GeV}$ for the subleading muon. The dielectron trigger requires the same thresholds of 17 and $8 \mathrm{GeV}$ on the $p_{\mathrm{T}}$ of the leading and subleading electron candidates, respectively. Additionally, the trigger requires that the electron candidates be isolated from other energy deposits in the calorimeter so an isolation requirement is imposed on the electron track.

Muons are reconstructed offline by a simultaneous fit of hits recorded in the silicon tracker and in the muon detectors [15]. Electrons are reconstructed from energy clusters in the ECAL and tracking information [14]. The two leading leptons are required to be of opposite electric charges and of the same flavor, with $p_{\mathrm{T}}>20 \mathrm{GeV}$ and $|\eta|<2.4$. For both candidates, a match with a corresponding trigger object is required. The dilepton invariant mass, $m_{\ell \ell}$, is required to satisfy $71 \mathrm{GeV}<m_{\ell \ell}<111 \mathrm{GeV}$. This will be referred to as the "Z boson mass window".

The particles in the event are reconstructed using the particle-flow $(\mathrm{PF})$ technique [34, 35], which consists of identifying each single particle with an optimized combination of all subdetector information. Depending on their signatures in the various subdetectors, particles fall into five different PF categories: muons, electrons, photons, neutral hadrons, and charged hadrons. The lepton candidates are required to be isolated from the other particles in the event, so to evaluate the isolation a scalar $p_{\mathrm{T}}$ sum of PF objects is calculated in the cone $\Delta R=\sqrt{(\Delta \phi)^{2}+(\Delta \eta)^{2}}$ around the direction of the object. The contribution from pileup to this isolation scalar $p_{\mathrm{T}}$ sum is subtracted using the average pileup energy per unit area in the $\eta-\phi$ plane evaluated for each event [36]. For electrons, the pileupsubtracted isolation sum is calculated in a cone of $\Delta R=0.3$ around the direction of the electron and is required to be below $15 \%$ of the electron $p_{\mathrm{T}}$. For muons, the radius is set to be $\Delta R=0.4$ and the isolation variable is required to be less than $12 \%$ of the muon $p_{\mathrm{T}}$. Lepton reconstruction, identification, and isolation efficiencies are measured using the "tag-and-probe" technique as described in ref. [37]. Efficiencies for simulated events are corrected using $\eta$ - and $p_{\mathrm{T}}$-dependent scale factors to account for differences between data and simulation. Scale factors typically range between 0.98 and 1.02 . 
The photons are reconstructed offline from energy clusters in ECAL [13]. Events for the $\gamma+$ jets processes are selected at the trigger level, where the presence of a high- $p_{\mathrm{T}}$ photon candidate is required. Since the instantaneous luminosity of the LHC increased during the data-taking period, the threshold in $p_{\mathrm{T}}$ increased as well, and the lower $p_{\mathrm{T}}$ threshold triggers are prescaled in order to keep the rate at a reasonable level. An unprescaled trigger is available only for a photon with transverse momentum $p_{\mathrm{T}}^{\gamma}>207 \mathrm{GeV}$. In order to further reduce the rate, a loose shower shape cutoff $\sigma_{\eta \eta}<0.24$ is imposed at trigger level, where $\sigma_{\eta \eta}$ measures the extension of the shower in pseudorapidity in terms of the energy-weighted spread within the $5 \times 5$ crystal matrix around the most energetic crystal in the photon cluster. For photon candidates, a match with a corresponding trigger object is required. For this analysis, only isolated high- $p_{\mathrm{T}}$ photons located inside the barrel region of the detector $(|\eta|<1.4)$ are considered. We concentrate on photons inside the barrel region because the data size of the templates, described in section 5.2, allows for a precise purity determination in this region. Around $40 \%$ of the photons convert into $\mathrm{e}^{+} \mathrm{e}^{-}$pairs inside the tracker material. Conversion track candidates are fitted from a combination of ECAL seeded tracks and Gaussian sum filter [38] electron tracks originate from a common vertex. The track pair is then matched to energy clusters in ECAL to identify a converted photon candidate. The final photon candidates are checked for possible overlap with electron candidates by looking for electron track seeds in the pixel detector or by using the characteristics of the track pair for converted photons. Isolation requirements are separately imposed on the pileupcorrected scalar $p_{\mathrm{T}}$ sum of neutral and charged hadrons, as well as on additional photons inside a cone of $\Delta R=0.3$ around the photon candidate direction. MC over data scale factors for the selection efficiencies of unconverted and converted photons without the electron veto are measured using the tag-and-probe technique on $\mathrm{Z} \rightarrow \mathrm{e}^{+} \mathrm{e}^{-}$events; the scale factors for the electron veto efficiency on signal photons are determined using $Z \rightarrow \mu^{+} \mu^{-} \gamma$ candidates. These scale factors range between 0.96 and 1.01 for photon candidates with $p_{\mathrm{T}}^{\gamma}>40 \mathrm{GeV}$.

Jets are reconstructed from the four-momentum vectors of all PF objects. The anti$k_{\mathrm{T}}$ clustering algorithm [39] is used here with a distance parameter of $R=0.5$ in its FASTJET [40] implementation. The jets are clustered by four-momentum summation. The reconstructed PF candidates are calibrated separately to account for the nonlinear and nonuniform response of the CMS hadron calorimeter, especially for neutral hadrons. Charged hadrons and photons are well measured in the silicon tracker and the ECAL, and therefore need only minimal corrections. Thus, the resulting jets require only small additional momentum adjustments. Jet energy corrections are obtained using GEANT4 simulated events generated with PYTHIA6. The energy contributions due to the presence of additional proton-proton interactions are subtracted from each jet using the measured pileup unit density in the event and the jet area [36]. The $\eta$ dependent corrections are adjusted using exclusive dijet events, while the $p_{\mathrm{T}}$-dependent corrections are adjusted using exclusive $\mathrm{Z}+1$-jet and $\gamma+1$-jet events in the data [41]. As a result of these adjustments, the reconstructed jets are corrected to the stable particle level [42]. For PF jets, the jet energy correction factor typically ranges from 1 to 1.2. Jets originating from pileup are rejected using the criteria described in ref. [43]. This rejects $90-95 \%$ of pure pileup jets while keeping over $99 \%$ of jets from the primary interaction. Jet identification quality re- 
quirements are imposed in order to remove spurious jets caused by noise in the calorimeter. The remaining jets are accepted for the analysis if they satisfy $p_{\mathrm{T}}>30 \mathrm{GeV}$ and $|\eta|<2.4$. Additionally, jets within a radius of $\Delta R<0.5$ with respect to the axes of each lepton or photon candidate are removed. This cut affects a small number of jets. For both $\mathrm{Z}+$ jets and $\gamma+$ jets selections, the presence of at least one jet is required.

The selection of $\mathrm{Z}+$ jets events is separate from the selection of $\gamma+$ jets events, and the two data samples are analyzed and corrected independently. The overlap between $\mathrm{Z}+$ jets and $\gamma+$ jets events is negligible. The analysis is repeated in four different, but not mutually exclusive, kinematic regions, with $p_{\mathrm{T}}^{V}>100 \mathrm{GeV}$ and

- $n_{\text {jets }} \geq 1$,

- $n_{\text {jets }} \geq 2$,

- $n_{\text {jets }} \geq 3$,

- $H_{\mathrm{T}}>300 \mathrm{GeV}$.

The rapidity of the $\mathrm{Z}$ boson is not restricted for the individual $\mathrm{Z}$ boson distributions. However, it is restricted to the rapidity range $|y|<1.4$ for the distributions of the ratio of $p_{\mathrm{T}}^{\mathrm{Z}}$ to $p_{\mathrm{T}}^{\gamma}$ because the photon is measured only in this central rapidity range. Rapidity is defined as $y=\frac{1}{2} \ln \left[\left(E+p_{z}\right) /\left(E-p_{z}\right)\right]$. The measured differential cross sections are binned in equal intervals of $\log _{10} p_{\mathrm{T}}(\mathrm{GeV})$ of width 0.045 from 100 to $800 \mathrm{GeV}$, corresponding to the overlap region between the $\mathrm{Z}+$ jets and $\gamma+$ jets phase space. This binning ensures that as the number of events decreases, the bin width increases in a regular way. In terms of the photon purity determination (defined in section 5.2), the bins are chosen such that there are enough events in all bins in the final distribution to ensure a reliable measurement.

\section{$5 \quad$ Background determination and unfolding}

\subsection{The $\mathrm{Z}+$ jets selection}

Events from the $\mathrm{Z}+$ jets process are selected as $\mathrm{Z} \rightarrow \mathrm{e}^{+} \mathrm{e}^{-}$and $\mathrm{Z} \rightarrow \mu^{+} \mu^{-}$candidates with one or more jets, as described in section 4 . The background-subtracted distributions are unfolded to the stable particle level for each decay channel separately and then combined.

Several SM processes contribute to backgrounds to the $\mathrm{Z}+$ jets signal. For low $p_{\mathrm{T}}^{\mathrm{Z}}$, the most important background is t $\overline{\mathrm{t}}$ production, whereas at higher $p_{\mathrm{T}}^{\mathrm{Z}}$ values, diboson production is the dominant background. Contributions due to $\mathrm{W}+$ jets and $\mathrm{WW}+$ jets are negligible for this analysis. The background contributions are subtracted using relative event rates predicted by MADGRAPH after an NNLO scaling for Drell-Yan and t⿹ samples and an NLO scaling for the electroweak backgrounds.

A cross check of the validity of the procedure for t $t \bar{t}$ background estimation is performed using an $\mathrm{e} \mu$ control sample in data. This sample is largely dominated by $\mathrm{t} \overline{\mathrm{t}}$ production with an additional contribution from fully leptonic $\mathrm{Z} \rightarrow \tau^{+} \tau^{-}$decays. Both in absolute scale and shape, the simulation reproduces the dilepton transverse momentum spectrum $\left(p_{\mathrm{T}}^{\ell \ell}\right)$ in the data within $10 \%$. This statement is valid both for a selection with a relaxed dilepton mass 
of $m_{\mathrm{e} \mu}>60 \mathrm{GeV}$ and a selection within the $\mathrm{Z}$ boson mass window as used for the final event selection. As a second check, the relative rate of e $\mu$ events in data and MC are compared to those of dielectron or dimuon events as a function of the dilepton $p_{\mathrm{T}}^{\ell \ell}$. Events with $\mathrm{e} \mu$ are selected by requiring the e $\mu$ invariant mass to be either in the $\mathrm{Z}$ boson mass window or in the whole mass range. Events from $\mathrm{e}^{+} \mathrm{e}^{-}$or $\mu^{+} \mu^{-}$are selected in the $\mathrm{Z}$ boson mass window. All four distributions of these relative event rates from simulation are compatible with data within $10 \%$. The $\mathrm{t} \overline{\mathrm{t}}$ background peaks at around $p_{\mathrm{T}}^{\ell \ell} \approx 100 \mathrm{GeV}$, where it amounts to $1.5 \%$ for the inclusive 1-jet selection and to $8 \%$ for the inclusive 3 -jet selection. In the high- $H_{\mathrm{T}}$ selection, it amounts to up to $12 \%$. For $H_{\mathrm{T}}>300 \mathrm{GeV}$, the relative rate drops below $0.5 \%$, while in the e $\mu$ channel no event is observed beyond $p_{\mathrm{T}}^{\mathrm{e}}>450 \mathrm{GeV}$.

At around $p_{\mathrm{T}}^{\ell \ell} \approx 150 \mathrm{GeV}$, the EW background increases and reaches a plateau of about $5-7 \%$ for all phase space selections of the analysis beyond $p_{\mathrm{T}}^{\ell \ell} \approx 400 \mathrm{GeV}$. The rate of the combined EW backgrounds predicted by simulation in a control region of multilepton final states is checked with data in the following way. Instead of selecting the two leading leptons, and then enforcing the same flavor requirement, we instead select the first two leptons matching the trigger objects with the same flavor. The rate of events gained with respect to the baseline selection is largely dominated by diboson events. These additional data events are compared to estimations from MADGRAPH, finding an agreement within roughly $10 \%$ for all jet multiplicity phase space selections. This comparison is done in the range $p_{\mathrm{T}}^{\ell \ell}<300 \mathrm{GeV}$.

The same selection criteria from data are used at the particle level: leading leptons are required to have $p_{\mathrm{T}}>20 \mathrm{GeV}$ and $|\eta|<2.4$, while jets are required to have $p_{\mathrm{T}}>30 \mathrm{GeV}$ within the region of $|\eta|<2.4$. The particle level jets in simulation are obtained by clustering the generated stable particles (after hadronization and including neutrinos) using the anti$k_{\mathrm{T}}$ algorithm with distance parameter of $R=0.5$. Electrons and muons have different energy losses due to final state radiation at particle level. In order to compensate for these differences, we define a "dressed" level to make the electron and muon channels compatible to within $1 \%$. This is achieved by defining in simulation a particle momentum vector by adding the momentum of the stable lepton and the momenta of all photons with a radius of $\Delta R=0.1$ around the stable lepton. All jets are required to be separated from each lepton by $\Delta R>0.5$.

The background-subtracted detector-level distributions from data are unfolded to the particle level. The unfolding response matrix includes detector resolution effects and efficiencies. We use MADGraPH to build a response matrix which allows us to map detectorlevel distributions to particle level. To quantify the bias introduced by the choice of the MC model, we use SHERPA as an alternative. The off-diagonal elements of the response matrices are small for both channels. For the dielectron channel, $85-95 \%$ of all events in a given bin of the reconstructed $p_{\mathrm{T}}^{\mathrm{ee}}$ distribution are mapped onto the same bin at the particle level. For the dimuon channel, at low $p_{\mathrm{T}}^{\mu \mu}$ around $85 \%$ fall in this category, whereas at very high $p_{\mathrm{T}}^{\mu \mu}$, only $67 \%$ stay in the same bin at the particle level. The remaining events typically fluctuate to directly neighboring bins. The iterative method used by d'Agostini [44], as implemented in the RooUnFolD package [45], is used to regularize the inversion of the matrix. Subsequently, the unfolded distributions from $\mathrm{Z} \rightarrow \mathrm{e}^{+} \mathrm{e}^{-}$and $\mathrm{Z} \rightarrow \mu^{+} \mu^{-}$are 
found to be compatible. They are combined using the best linear unbiased estimator [46] to obtain the final distributions. The resulting averaged leptonic $\mathrm{Z}+$ jets distributions from both channels are not corrected to the total cross section.

\subsection{The $\gamma+$ jets selection}

After selecting $\gamma+$ jets events (as described in section 4), the photon signal purity is determined in each $p_{\mathrm{T}}$ bin. The main background is due to QCD multijet production, where either one of the jets, or an electron or $\pi^{0}$ inside a jet, is misidentified as a photon candidate. Since simulations do not provide a reliable description of this background, the purity, which is defined as the number of true isolated photons from the hard scattering versus the number of all photon candidates, is determined from data. At the particle level, a true isolated photon is defined as a prompt photon, around which the scalar sum of the $p_{\mathrm{T}}$ of all stable particles in a cone of radius $\Delta R=0.4$ is less than $5 \mathrm{GeV}$. Similarly, at the detector level, for each $p_{\mathrm{T}}$ bin of the photon spectrum, the purity is determined through a fit of the photon isolation sum variable $I_{\mathrm{ph}}^{\mathrm{PF}}$, defined as the scalar $p_{\mathrm{T}}$ sum of all other PF photons around the axis of the selected photon candidate, inside a cone of $\Delta R=0.4$. The sum is corrected for the pileup contribution and the energy deposit ("footprint") of the selected photon candidate itself. The $I_{\mathrm{ph}}^{\mathrm{PF}}$ distribution for the data is fitted as a sum of signal and background template distributions, in each $p_{\mathrm{T}}$ bin, in order to calculate the purity $f: I_{\mathrm{ph}}^{\mathrm{PF}}($ data $)=f I_{\mathrm{ph}}^{\mathrm{PF}}($ signal $)+(1-f) I_{\mathrm{ph}}^{\mathrm{PF}}($ background $)$.

In order to model the contribution of the underlying event to the photon component isolation sum around the signal photon candidate, a signal template is obtained from the data through the random-cone (RC) method [47]. After selecting photon candidates fulfilling a requirement on the shower shape of $\sigma_{\eta \eta}<0.011$, a cone of $\Delta R=0.4$ is randomly chosen in $\phi$ at the same $\eta$ as the photon candidate, excluding the back-to-back direction to avoid selecting any recoiling jet. The candidate cone is rejected if it contains objects originating from a hard interaction, e.g., jets with $p_{\mathrm{T}}>30 \mathrm{GeV}$ or photons with $p_{\mathrm{T}}>$ $20 \mathrm{GeV}$. The RC templates show a very good agreement over orders of magnitude between data, simulation, and the true-photon MC templates obtained by matching a detector-level photon candidate with an isolated photon at the particle level. Background templates are constructed by selecting photon candidates in the data with an inverted shower shape requirement, $0.011<\sigma_{\eta \eta}<0.014$. Since there are a small number of background events with high $p_{\mathrm{T}}$ photon candidates, the templates are obtained in wider bins of $p_{\mathrm{T}}^{\gamma}$ than used in the final analysis. After construction of the templates, a binned maximum-likelihood fit to the $I_{\mathrm{ph}}^{\mathrm{PF}}$ data distributions is performed as a sum of the signal and background template distributions. The statistical uncertainty on the fit includes the effect of the limited template sample size. An example of the fit for the photon component of the photon isolation can be seen in figure 1 (left). The results of such fits are displayed in figure 1 (right), where the black dots represent the measured purity and the solid lines represent the statistical uncertainties including the template uncertainties. The purity ranges from around $65 \%$ in the lower $p_{\mathrm{T}}$ bins to $90 \%$ at high $p_{\mathrm{T}}$. The shaded band represents the total statistical and systematic uncertainties in the purity measurement. The difference in the fractions obtained from the closure test on simulation is treated as 

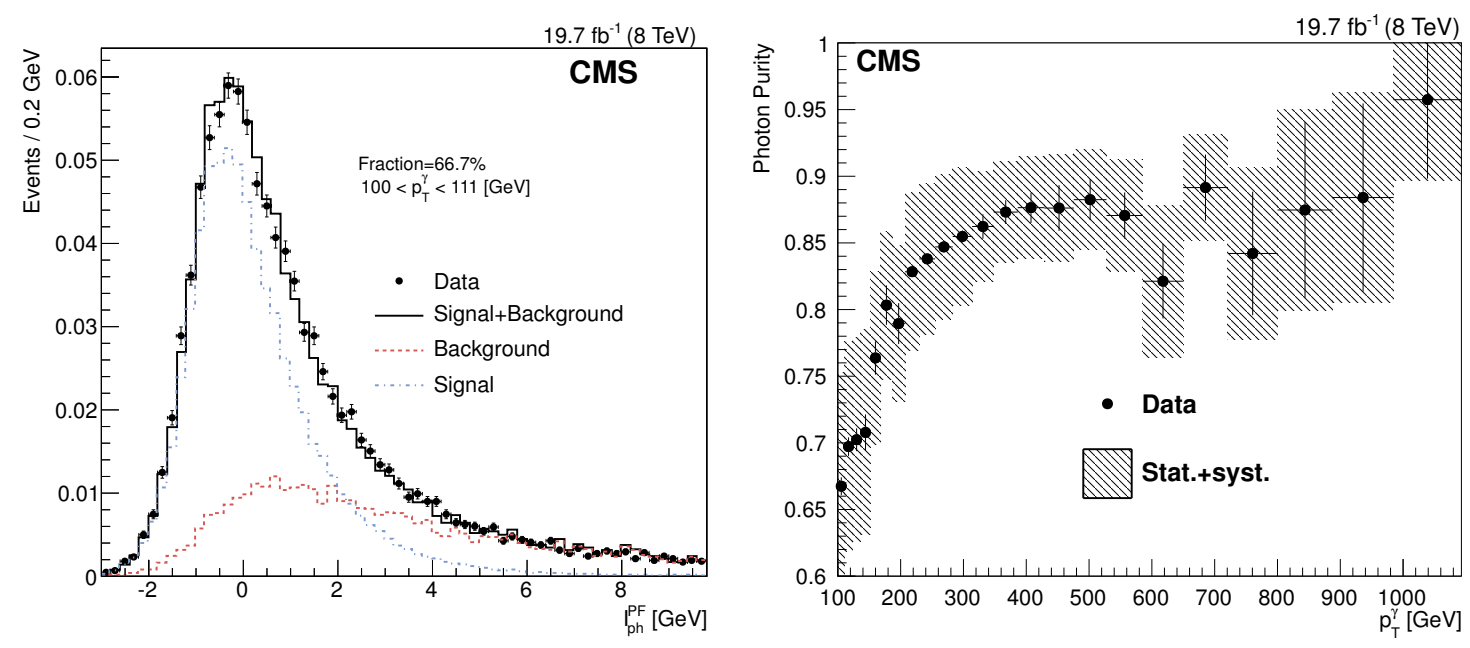

Figure 1. The purity fit on the photon component of the photon isolation $I_{\mathrm{ph}}^{\mathrm{PF}}$ in the photon transverse momentum bin between 100 and $111 \mathrm{GeV}$ in data (left). The photon purity as function of the photon transverse momentum (right). The dots are the data points, the dot-dashed line is the signal template, and the dotted line represents the background component. The solid line represents the fit and the legend shows the resulting purity fraction of $66.7 \%$.

the systematic uncertainty. True MC signal (background) templates are determined using identified photons at detector level matched (not matched) to a particle-level photon. The closure test uses the same approach for deriving templates that was used in data: it takes simulated samples and compares the resulting templates of the data-driven approach with the templates constructed exploiting the MC-truth information. This is the dominant source of systematic uncertainty in the purity estimate. Other effects, such as a change in the $\sigma_{\eta \eta}$ requirement, are found to be negligible. The systematic uncertainties of the purity estimate are discussed further in section 6 . The data yields in each $p_{\mathrm{T}}$ bin, after correcting for purity, are unfolded to the particle level with a procedure identical to the one used for the $\mathrm{Z}+$ jets process. Over the whole $p_{\mathrm{T}}^{\gamma}$ spectrum, the diagonal elements of the response matrix contain more than $90 \%$ of the events.

\section{$6 \quad$ Systematic uncertainties}

For $\mathrm{Z}+$ jets and $\gamma+$ jets we consider the following uncertainties: the jet energy scale (JES) uncertainty, the jet energy resolution (JER) uncertainty, uncertainties due to the MC model from the unfolding procedure (UF), the pileup uncertainty (PU), and the luminosity uncertainty (Lumi). Systematic effects specific to $\gamma+$ jets are those related to the photon energy scale $(\gamma$ ES) and the purity determination ( $\gamma$ Pur). For Z + jets, we consider the background subtraction (BG) and the lepton (muon and electron) momentum scale (LS) and resolution (LRES) uncertainties, as well as uncertainties in the lepton efficiency and isolation. We also consider lepton efficiency scale factors (lep SFs) for the $\mathrm{Z}+$ jets events. For both processes, the uncertainty associated with the luminosity is a flat $2.6 \%$ over the whole range [48]. 
The systematic effects due to scale uncertainties affect the data, so we vary the momenta of the jets or the leptons independently within their uncertainties and rerun the unfolding on the shifted distribution. The differences in the final results are taken to be the systematic uncertainties.

The uncertainty due to the JES affects distributions through the jet $p_{\mathrm{T}}$ threshold [41]. For central jets, the JES uncertainty is around $3 \%$ at $30 \mathrm{GeV}$, decreasing to $1 \%$ at $100 \mathrm{GeV}$. Therefore, in the $n_{\text {jets }}=1$ case, the boson $p_{\mathrm{T}}$ is almost completely unaffected. For the 2or 3-jet inclusive phase space selection, the requirement of additional jet activity increases the uncertainty in the photon and Z spectra due to JES to $5-10 \%$ over the whole $p_{\mathrm{T}}^{V}$ range. In the $H_{\mathrm{T}}>300 \mathrm{GeV}$ selection, the JES uncertainty is around $5-7 \%$ at low $p_{\mathrm{T}}^{V}$ and below $1 \%$ for $p_{\mathrm{T}}^{V}>400 \mathrm{GeV}$.

Systematic effects due to purity and background subtraction have to be applied prior to unfolding to evaluate the uncertainty. The other uncertainties affect the response matrix in the simulation, and the unfolding is performed with these modified matrices to determine the relative uncertainty. For example, we modify the resolution of jets in the MC and then calculate a new response matrix with these modified resolutions. The difference between this result and the nominal result is taken as the uncertainty due to JER.

For the $\mathrm{Z}+$ jets process, the dominant sources of uncertainty are the lepton SFs and the LS in the $n_{\text {jets }} \geq 1$ case and the JES uncertainty otherwise. The uncertainty due to the background subtraction is typically below $1 \%$. The lepton resolution uncertainty has an effect that is typically less than $0.5 \%$. The effect of the electron energy scale uncertainty increases with $p_{\mathrm{T}}^{\mathrm{Z}}$ from $1 \%$ at $40 \mathrm{GeV}$ to $5 \%$ at $800 \mathrm{GeV}$. For muons, the scale uncertainty has an effect $<1 \%$ up to $250 \mathrm{GeV}$, which increases up to $15 \%$ at high $p_{\mathrm{T}}^{\mathrm{Z}}$. Above $200 \mathrm{GeV}$, the track becomes very straight, and so the influence of the muon system becomes more relevant with respect to the tracker for the muon $p_{\mathrm{T}}$ distribution. This leads to an increase in the muon scale uncertainty.

For the unfolding procedure, an additional check using the matrix obtained from SHERPA instead of that from MADGRAPH is performed, resulting in a cross section uncertainty of $2-3 \%$ for all phase space regions. A cross check using the Singular Value Decomposition regularization method [49] for the unfolding shows negligible deviations.

The JER is measured to be about $5 \%$ larger than predicted in simulation for the central detector part $\left(\left|\eta^{\mathrm{jet}}\right|<1.4\right)$ with an uncertainty of about $5 \%$, and roughly $10 \%$ larger in the endcaps with an uncertainty of roughly $7 \%$ [41]. The JER and pileup uncertainties in $\mathrm{Z}+$ jets events have values typically below $1 \%$. The uncertainties on $\mathrm{Z}+$ jets are summarized in table 1.

Aside from the $p_{\mathrm{T}}$ spectrum, we also consider two additional variables for the $\mathrm{Z}+$ jets final state: the ratio of the $p_{\mathrm{T}}^{\mathrm{Z}}$ to $H_{\mathrm{T}}$ and to the $\log _{10}\left(p_{\mathrm{T}}^{\mathrm{Z}} / p_{\mathrm{T}}^{\mathrm{j} 1}\right)$, where $p_{\mathrm{T}}^{\mathrm{j} 1}$ is the transverse momentum of the largest jet in the event. Most uncertainties in these distributions are similar to those described above for the $p_{\mathrm{T}}^{\mathrm{Z}}$ spectra, with the exception of the JES uncertainty. The latter has a larger influence on hadronic quantities $\left(H_{\mathrm{T}}, p_{\mathrm{T}}\right.$ of the jets, and $\left.n_{\text {jets }}\right)$ which enter the distributions directly, rather than through phase space selections.

For the $\gamma+$ jets process, the dominant uncertainty is due to the photon purity. This is a result of the difference between the shapes of the templates defined using the data-driven 


\begin{tabular}{|l|cccccccc|}
\hline Process & $\begin{array}{c}\text { JES } \\
\left(n_{\text {jets }} \geq 1\right)\end{array}$ & $\begin{array}{c}\text { JES } \\
\text { (otherwise) }\end{array}$ & JER & Lep SFs & UF & $\begin{array}{c}\text { PU, BG } \\
\text { LRES }\end{array}$ & LS & Lumi \\
\hline $\mathrm{Z} \rightarrow \mathrm{e}^{+} \mathrm{e}^{-}$ & $1-3 \%$ & $5-10 \%$ & $<1 \%$ & $3-4 \%$ & $2-3 \%$ & $<1 \%$ & $1-5 \%$ & $2.6 \%$ \\
$\mathrm{Z} \rightarrow \mu^{+} \mu^{-}$ & $1-3 \%$ & $5-10 \%$ & $<1 \%$ & $2.5-5.5 \%$ & $2-3 \%$ & $<1 \%$ & $<1 \%$ & $2.6 \%$ \\
\hline
\end{tabular}

Table 1. Systematic Uncertainties for the $p_{T}^{Z}$ Spectrum.

\begin{tabular}{|l|cccccccc|}
\hline Process & $\begin{array}{c}\text { JES } \\
\left(n_{\text {jets }} \geq 1\right)\end{array}$ & $\begin{array}{c}\text { JES } \\
(\text { otherwise })\end{array}$ & JER & UF & PU & $\gamma$ Pur & $\gamma$ ES & Lumi \\
\hline$\gamma$ & $1-3 \%$ & $5-10 \%$ & $0.5-1.5 \%$ & $2 \%$ & $<0.5 \%$ & $4-10 \%$ & $3 \%$ & $2.6 \%$ \\
\hline
\end{tabular}

Table 2. Systematic Uncertainties for the $p_{\mathrm{T}}^{\gamma}$ Spectrum.

techniques from above and the distributions of the "true" templates for isolated photons in simulated events. Data samples are generated using the distributions of isolation variables for every bin of each variable with the fractions measured in data. Each of these is fitted with templates built in MC using the same techniques as on data, and the average difference between these fitted fractions and the generated fractions is quoted as the systematic uncertainty due to photon purity estimation [47]. This difference is around $10 \%$ when $p_{\mathrm{T}}^{\gamma} \approx 100 \mathrm{GeV}$ and it decreases to roughly $4 \%$ at $p_{\mathrm{T}}^{\gamma} \approx 400 \mathrm{GeV}$ for the inclusive $n_{\text {jets }} \geq 1$ selection. A change in the selection criteria on $\sigma_{\eta \eta}$ leads to negligible effects on the purity estimation. The background templates do not show any dependence on $H_{\mathrm{T}}$ or on the number of jets in the analysis. Therefore, the same background templates of the inclusive selection are used for all phase space regions and a similar template uncertainty is obtained.

The JER uncertainty has a negligible effect in the analysis region $p_{\mathrm{T}}^{\gamma}>100 \mathrm{GeV}$. For the 2- or 3-jet phase space and high $H_{\mathrm{T}}>300 \mathrm{GeV}$ selection, the resolution uncertainty has an effect around $0.5-1.5 \%$. The effect of the $\gamma$ ES uncertainty on the cross section measurement is constant across the whole range and less than $3 \%$. The unfolding uncertainty is estimated by using an unfolding matrix from PYTHIA6 simulation and is around $2 \%$. The uncertainty in the pileup interactions is evaluated by rescaling the cross section of minimum bias events by $5 \%$ in the $\mathrm{MC}$ reweighting procedure. Typically, these uncertainties are very small, below $0.5 \%$. These systematic uncertainties for the $\gamma+$ jets process are summarized in table 2 .

\section{Results}

\subsection{Differential cross sections}

In figures 2 and 3 , we present the measured differential cross sections as functions of the $p_{\mathrm{T}}^{\mathrm{Z}}$ and the $p_{\mathrm{T}}^{\gamma}$ for two selections of $\mathrm{Z}+$ jets and $\gamma+$ jets events $\left(n_{\text {jets }} \geq 1\right.$ and $\left.n_{\text {jets }} \geq 2\right)$ and compare them with estimates from BLACKHAT and MADGRAPH+PYTHIA6. In figure 4, we present the ratio of the inclusive 2-jet events to the inclusive 1 -jet events. For $\mathrm{Z}+$ jets, we also compare the data to SHERPA results. The NLO BLACKHAT estimate is corrected for nonperturbative effects (hadronization and underlying event) using MADGRAPH+PYTHIA6. 
These corrections are typically around $2 \%$. We use the $n$-jets BLACKHAT sample for comparison with data and other $\mathrm{MC}$ generators in the corresponding inclusive $n$-jets selection. The $\mathrm{Z}+$ jets simulations from SHERPA and MADGRAPH+PYTHIA6 are rescaled by a constant NNLO $K$-factor of $K=1.197$, as calculated with FEWZ 3.1 [22], while for $\gamma+$ jets the LO cross section from MADGRAPH+PYTHIA6 is used as no NNLO $K$-factor is available for $\gamma+$ jets. In all figures, the hatched band surrounding the data points represents the total uncertainty in the measurement, while the error bars show the statistical uncertainty. Similarly, in the MC/data ratio plots, the error bars around the points centered at one represent the relative statistical uncertainties on the data, while the hatched band represents the relative total uncertainty of statistics and systematics on the data. The shaded bands around the MC simulation/data ratios for MADGRAPH+PYTHIA6 and SHERPA represent the statistical uncertainty (stat. unc.) in the simulation. The outer hatched band around the BLACKHAT/data ratio (using MSTW2008) shows the total uncertainty of the estimate due to PDF and scale variations, while the inner hatched band indicates the uncertainty due to the variations within the MSTW2008 eigenvector set [50]. Analogous variations using the CT10 and NNPDF2.3 PDF sets lead to similar uncertainties. Not shown in the figures is the statistical uncertainty for the BLACKHAT calculations that amounts to less than $1-3 \%$ for $n_{\text {jets }} \geq 1,2$ and to $5-10 \%$ for $n_{\text {jets }} \geq 3$ in the $p_{\mathrm{T}}^{Z}$ spectra. In the distributions of the observables $p_{\mathrm{T}}^{\mathrm{Z}} / H_{\mathrm{T}}$ and $\log _{10}\left(p_{\mathrm{T}}^{\mathrm{Z}} / p_{\mathrm{T}}^{\mathrm{j} 1}\right)$, the statistical uncertainty is $6 \%$ except in the tails where there are fewer events. In the $p_{\mathrm{T}}^{\gamma}$ spectra, the statistical uncertainty is $3-5 \%$ in the $n_{\text {jets }} \geq 1$ case and $4-10 \%$ in the $n_{\text {jets }} \geq 2$ and 3 cases. The fluctuations seen in the BLACKHAT distributions between adjacent bins are statistical in nature. Overlaid are BLACKHAT estimates using the NNPDF (dashed) and CT10 (dotted) PDF sets.

In the $\mathrm{Z}+$ jets distributions for both phase space selections $\left(n_{\text {jets }} \geq 1\right.$ and $n_{\text {jets }} \geq 2$, figures 2 and 3), we observe the same qualitative behavior of the ratio of the MADGraPH+PYThia6 simulation to data, which is flat about unity up to around 150-200 GeV and then increases to about 1.3 at higher $p_{\mathrm{T}}$. Estimates from SHERPA are lower than the data for $p_{\mathrm{T}}^{\mathrm{Z}}<50 \mathrm{GeV}$, while for higher $p_{\mathrm{T}}^{\mathrm{Z}}$ they increase to around $20 \%$ higher. In the $n_{\text {jets }} \geq 1$ case, BLACKHAT shows a flat ratio with respect to data starting around $p_{\mathrm{T}}^{\mathrm{Z}} \approx 100 \mathrm{GeV}$, but underestimates the yield seen in data by $8-10 \%$, whereas in the $n_{\text {jets }} \geq 2$ case, BlackHat agrees with the data within the uncertainties for the whole range. For all multiplicity phase space selections, the systematic uncertainty in the MSTW2008 PDF set is $2-3 \%$ in the BlaCKHAT estimate. The central points of CT10 show a difference compared to MSTW2008 of at most $4 \%$, whereas NNPDF shows a variation of $2 \%$. The scale uncertainty for MSTW2008 in the BLACKHAT estimate, as determined through independent variations of the renormalization and factorization scales by factors of 2 and 0.5 , leads to an envelope with values of typically $5-10 \%$.

In the $n_{\text {jets }} \geq 2$ and $n_{\text {jets }} \geq 1 \gamma+$ jets case, BlACKHAT reproduces the shape of the data distribution, but underestimates the rate by approximately $10-15 \%$ throughout most of the range.

In figure 4, we see that the inclusive 1-jet over inclusive 2-jet $p_{\mathrm{T}}^{\mathrm{Z}}$ cross section ratio increases until a plateau is reached at around $350 \mathrm{GeV}$. The systematic uncertainties are 
treated as fully correlated in the ratio. The distributions are well predicted by MADGRAPH+PYTHIA6 in both channels. SHERPA underestimates the relative rate of inclusive 2-jets events. For BlackHat, the inclusive 2-jet generated sample is used to predict the 2 -jet rate and to compute the ratio with the predicted rates from the inclusive 1-jet sample; BlackHat overestimates the ratio by $10 \%$ for $p_{\mathrm{T}}^{\mathrm{Z}}>100 \mathrm{GeV}$ in both the $\gamma+$ jets and $\mathrm{Z}+$ jets cases.

For $\mathrm{Z}+$ jets, we study the variables $p_{\mathrm{T}}^{\mathrm{Z}} / H_{\mathrm{T}}$, shown in figure 5 , and $\log _{10}\left(p_{\mathrm{T}}^{\mathrm{Z}} / p_{\mathrm{T}}^{\mathrm{j} 1}\right)$, shown in figure 6 , which allow us to test the validity of NLO estimations. In particular, we examine these distributions as quantities where NLO estimations might reach their calculational limit due to large logarithms or where missing higher-order effects could play a larger role.

For events which contain a dominant high- $p_{\mathrm{T}}$ jet, $p_{\mathrm{T}}^{\mathrm{Z}} / H_{\mathrm{T}}$ tends to unity as the jet carries most of the $p_{\mathrm{T}}$ of the event. Events that populate the high-end tail of the distribution have either additional jets outside of the acceptance in the forward region or additional hadronic radiation that is not clustered in jets with $p_{\mathrm{T}}^{\text {jet }}>30 \mathrm{GeV}$. In hadronic searches for new physics, these events contribute to signatures with a high $\not_{\mathrm{T}} / H_{\mathrm{T}}$ ratio. Almost all events with two or more jets inside the jet acceptance selections have $p_{\mathrm{T}}^{\mathrm{Z}} / H_{\mathrm{T}}$ values below one. This behavior can be observed in figure 5: increasing the number of required jets leads to a shift of the complete distribution towards lower values. The nonperturbative corrections are slightly larger, typically below $5 \%$ in the bulk of the distribution, reaching $10 \%$ in the tails for all variables examined here. Overall, MADGRAPH+PYTHIA6 predicts the rate and shape best up to the tails, while SHERPA shows differences in both shape and rates. The BLACKHAT generator performs well for the bulk of the distribution, but fails to reproduce the tails. This is especially evident in the high-end tail of the distribution, where we see a sharp drop in the ratio of BLACKHAT simulations to data. In this portion of phase space, BLACKHAT is effectively reduced from an NLO to LO calculation as the $n+1$ jet LO calculation in the inclusive $n$ jet case dominates here, whereas the other portions provide negligible contributions. This feature is also confirmed by the sharp increase of scale uncertainties in BLACKHAT estimates, which have a step-like increase from below $10 \%$ to around $60 \%$ at this point. Therefore, this sharp change in the BLACKHAT over data ratio (e.g., around 1.2 and 1.1 in figure 5) is expected and indicates the "boundary" between the regions where a fixed-order calculation gives a suitable estimation and where we would need the parton showering to add soft jets or jets in the forward regions of the detector. Additionally, we can use this to check for any large logarithmic contributions in the lower end of this $p_{\mathrm{T}}^{\mathrm{Z}} / H_{\mathrm{T}}$ distribution. We see from the agreement in both the 2- and 3 -jet cases that there is no evidence of any such contributions.

The distribution of the second variable, $\log _{10}\left(p_{\mathrm{T}}^{\mathrm{Z}} / p_{\mathrm{T}}^{\mathrm{j} 1}\right)$, shown in figure 6 , shows similar behavior. For events with exactly one jet, the $\mathrm{Z}$ boson and the jet are back-to-back, with $p_{\mathrm{T}}^{\mathrm{Z}} \approx p_{\mathrm{T}}^{\mathrm{j} 1}$, and the distribution peaks around zero. Events where the $\mathrm{Z}$ boson is the dominating object will have positive values. If the $\mathrm{Z}$ boson carries less $p_{\mathrm{T}}$ than most of the jets, the variable has negative values. With increasing jet multiplicity the distribution still peaks around zero, but broadens. Figure 6 shows a comparison of MC estimates to data, which is unfolded to particle level. The MADGRAPH+PYTHIA6 calculation performs well in estimating the behavior of the data for all inclusive multiplicty selections, but there is a 

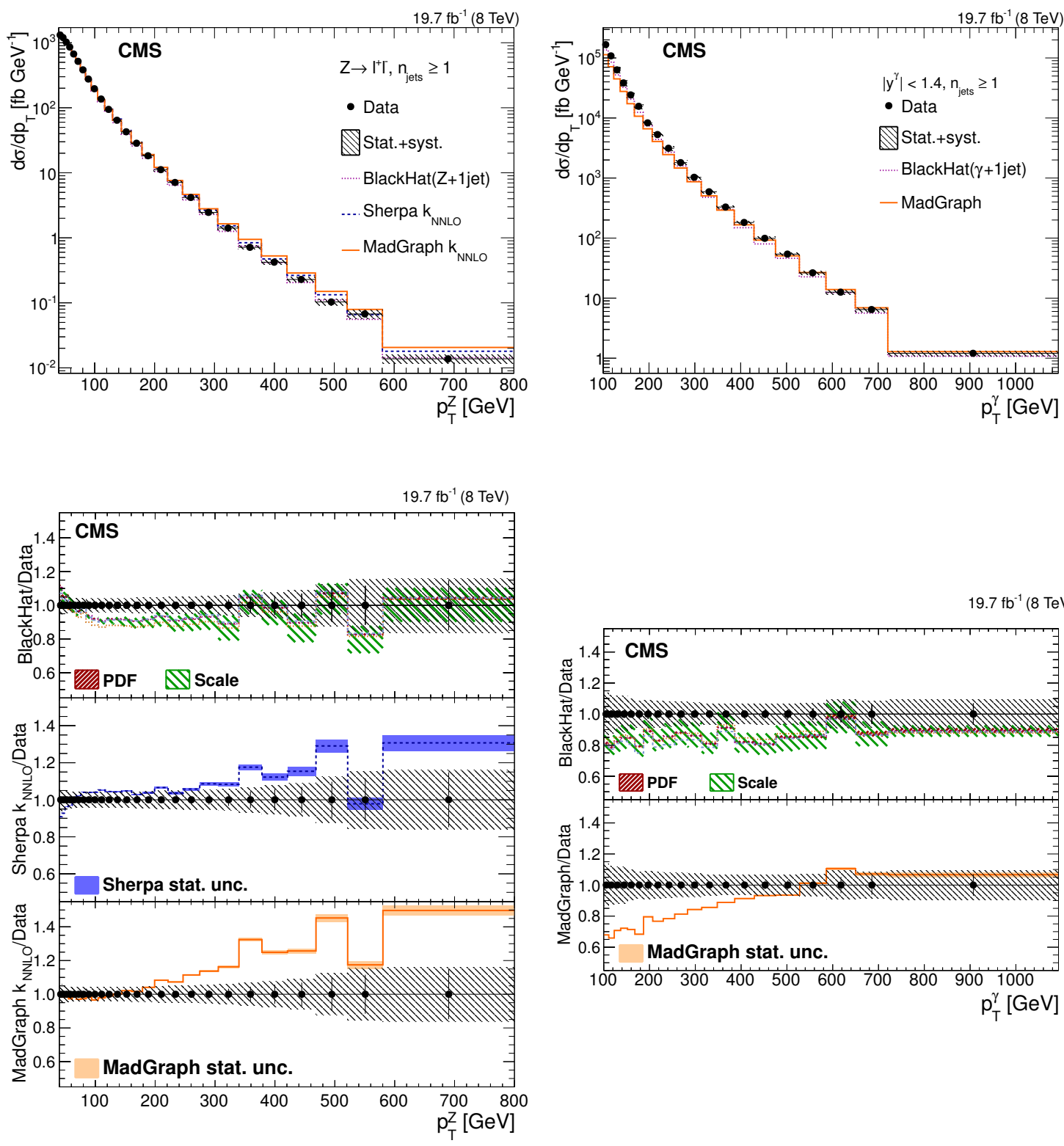

$19.7 \mathrm{fb}^{-1}(8 \mathrm{TeV})$

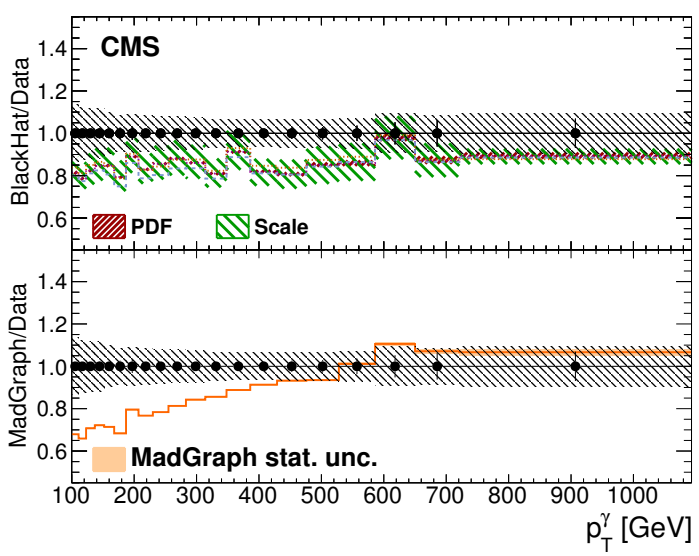

Figure 2. Top left: differential cross section for $\mathrm{Z}$ boson production as a function of $p_{\mathrm{T}}^{\mathrm{Z}}$ for an inclusive $\mathrm{Z}+$ jets, $n_{\text {jets }} \geq 1$ selection of detector-corrected data in comparison with estimations from MADGraph+PYTHIA6, ShERPa, and BlackHat. Top right: differential cross section for photon production as a function of $p_{\mathrm{T}}^{\gamma}$ for an inclusive $\gamma+$ jets, $n_{\text {jets }} \geq 1$ selection for central rapidities $\left|y^{\gamma}\right|<1.4$ in detector-corrected data is compared with estimations from MADGRAPH+PYTHIA6 and BLACKHAт. A detailed explanation is given in section 7.1. The bottom plots give the ratio of the various theoretical estimations to the data in the $\mathrm{Z}+$ jets case (bottom left) and $\gamma+$ jets case (bottom right). 

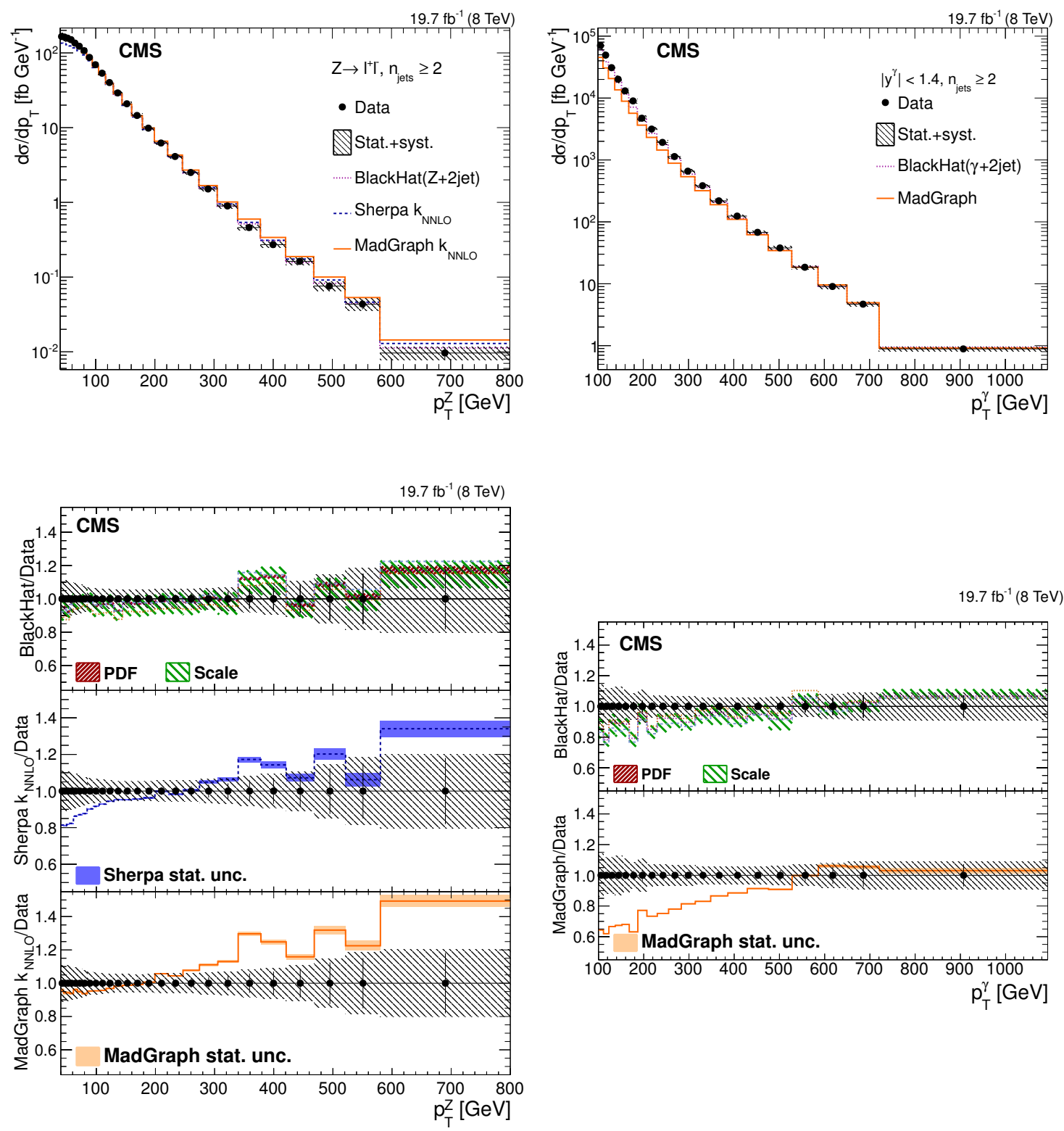

Figure 3. Top left: differential cross section for $\mathrm{Z}$ boson production as a function of $p_{\mathrm{T}}^{\mathrm{Z}}$ for an inclusive $\mathrm{Z}+$ jets, $n_{\text {jets }} \geq 2$ selection of detector-corrected data in comparison with estimations from MADGraph+PYThia6, SherPA, and BlackHAT. Top right: differential cross section for photon production as a function of $p_{\mathrm{T}}^{\gamma}$ for an inclusive $\gamma+$ jets, $n_{\text {jets }} \geq 2$ selection for central rapidities $\left|y^{\gamma}\right|<1.4$ in detector-corrected data is compared with estimations from MADGRAPH+PYTHIA6 and BLACKHAT. A detailed explanation is given in section 7.1. The bottom plots give the ratio of the various theoretical estimations to the data in the $\mathrm{Z}+$ jets case (bottom left) and $\gamma+$ jets case (bottom right). 

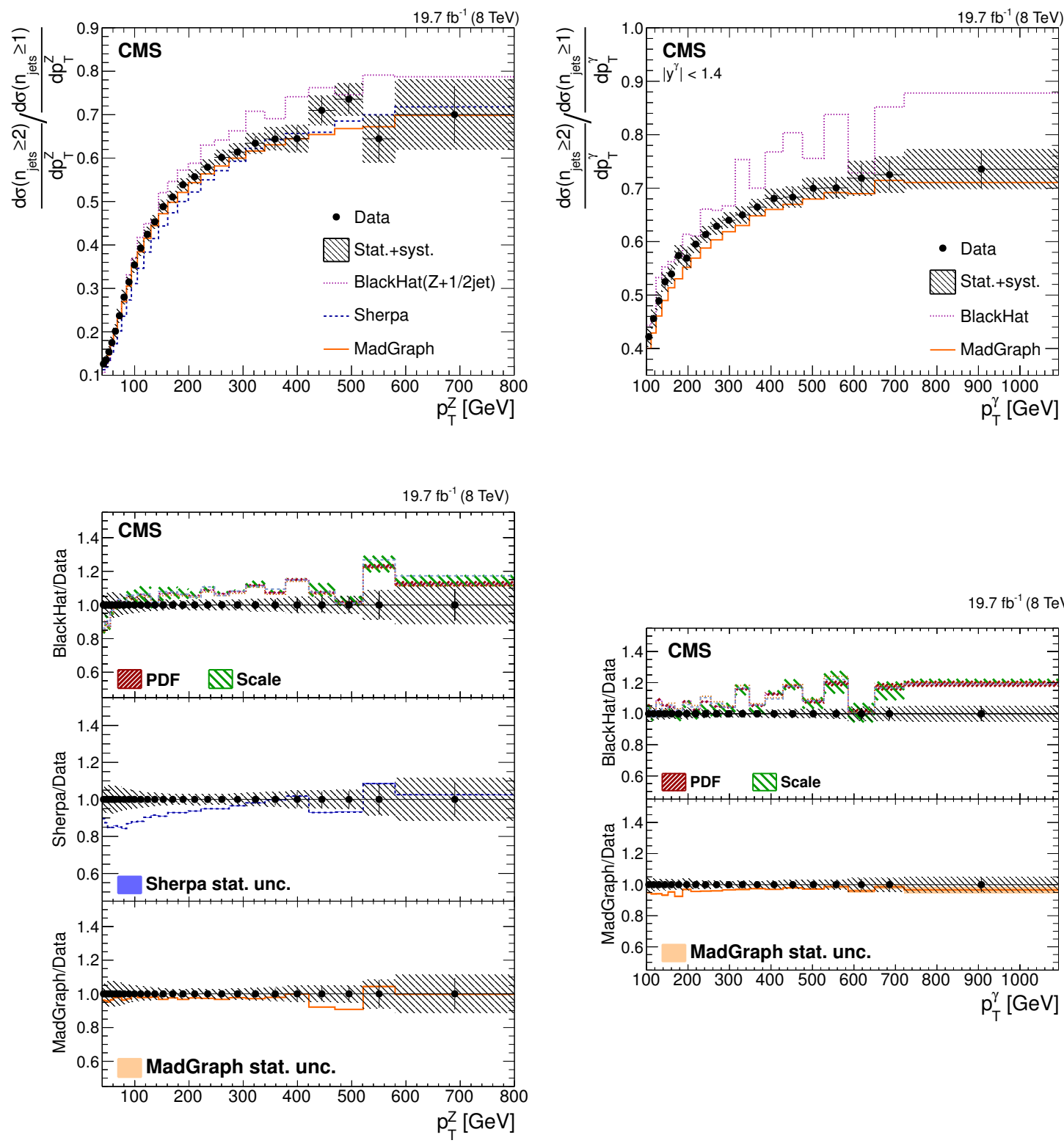

$19.7 \mathrm{fb}^{-1}(8 \mathrm{TeV})$

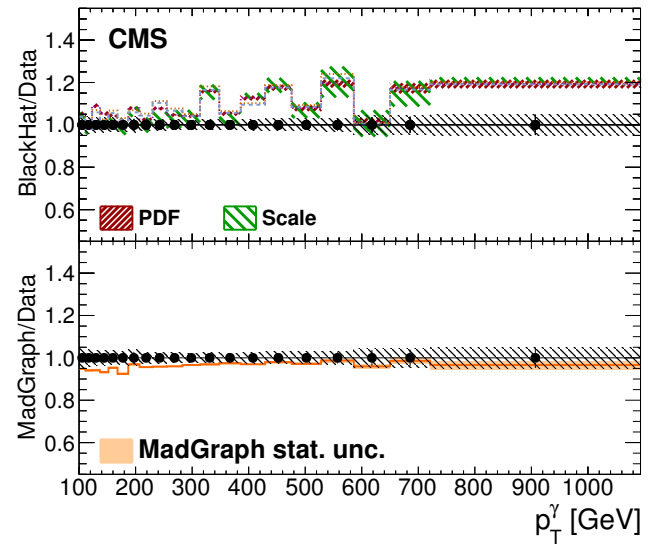

Figure 4. Ratio of the inclusive rates for $n_{\text {jets }} \geq 2$ and $n_{\text {jets }} \geq 1$ versus the transverse momentum of the boson for $\mathrm{Z}+$ jets in detector-corrected data compared to estimations from MADGRAPH+PYTHIA6, SHERPA, and BLACKHAT (top left) and for $\gamma+$ jets for central rapidities $\left|y^{\gamma}\right|<1.4$ in detector-corrected data compared with estimations from MADGRAPH+PYTHIA6 and BLACKHAT (top right). A detailed explanation is given in section 7.1. The bottom plots give the ratio of the various theoretical estimations to the data in the $\mathrm{Z}+$ jets case (bottom left) and $\gamma+$ jets case (bottom right). 

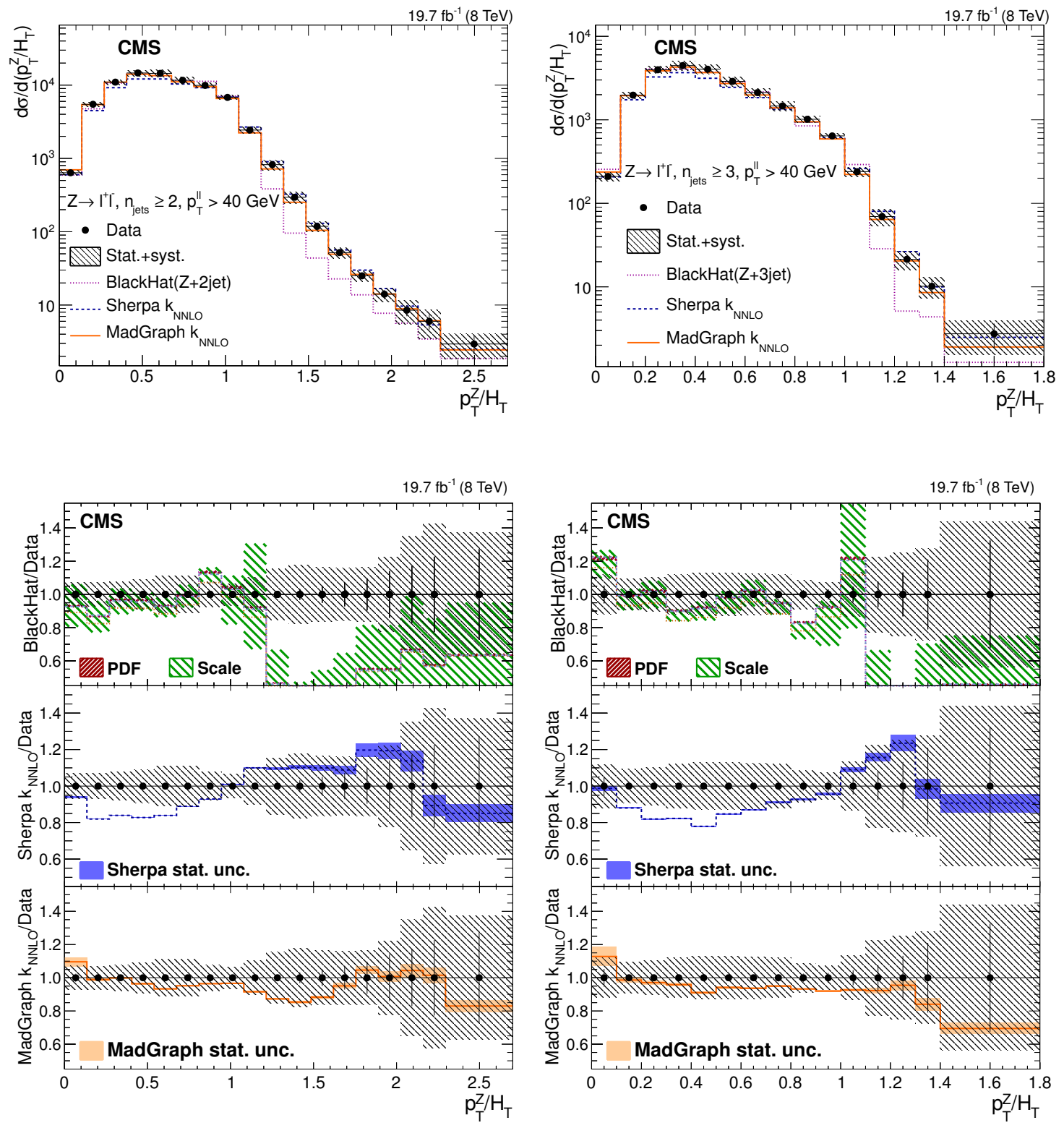

Figure 5. The measured distribution of the observable $p_{\mathrm{T}}^{\mathrm{Z}} / H_{\mathrm{T}}$ ratio for $n_{\text {jets }} \geq 2$ (top left) and $n_{\text {jets }} \geq 3$ (top right) for $\mathrm{Z}+$ jets in detector-corrected data compared with estimations from MadGraph+Pythia6, Sherpa, and BlackHat. A detailed explanation is given in section 7.1. The bottom plots give the ratio of the various theoretical estimations to the data in the $n_{\text {jets }} \geq 2$ case (bottom left) and $n_{\text {jets }} \geq 3$ case (bottom right). 
slope within uncertainties in the MC/data plot. On the other hand, BLACKHAT performs well in the middle range, but the behavior in the tails indicates that jet production due to higher-order diagrams is missing. In the $\mathrm{Z}+$ jets, $n_{\text {jets }} \geq 2$ phase space we observe a drop at the value $\log _{10}\left(p_{\mathrm{T}}^{\mathrm{Z}} / p_{\mathrm{T}}^{\mathrm{j} 1}\right)=0.3 \approx \log _{10}(2)$, corresponding to $p_{\mathrm{T}}^{\mathrm{j} 1} \approx p_{\mathrm{T}}^{\mathrm{j} 2}$ with both jets recoiling against the $\mathrm{Z}$ boson direction. The distribution drops at the point where the third-leading jet becomes relevant. Since we use the inclusive 2-jet BlackHat sample in that phase space, 3-jet events are only available as LO contributions in the real part. Therefore, the estimation is effectively an LO calculation at that point onwards, and subsequently becomes less precise and the scale variation uncertainty increases to around $30 \%$ at that point.

\subsection{The $\mathrm{Z} / \gamma$ ratio}

In order to compare the cross sections for $\mathrm{Z}+$ jets and $\gamma+$ jets, the rapidity range of the bosons is restricted to $\left|y^{V}\right|<1.4$ because this is the selected kinematic region for the photons. The ratio of the differential cross sections as a function of $p_{\mathrm{T}}$ is measured in the four phase space regions: $n_{\text {jets }} \geq 1,2,3$, and $H_{\mathrm{T}}>300 \mathrm{GeV}, n_{\text {jets }} \geq 1$.

Statistical uncertainties in the ratio are propagated using the diagonal terms of the covariance matrices. The sources of systematic uncertainty such as the JES, the luminosity uncertainty, and the JER are correlated between $\mathrm{Z}+$ jets and $\gamma+$ jets and therefore cancel in the ratio. The remaining uncertainties are results of the photon purity measurement, unfolding uncertainty, the uncertainties in the efficiency determination for photons, and the lepton energy or momentum scale uncertainty.

The resulting ratio distributions are shown in figures 7 and 8 for all selections. The $\mathrm{Z}+$ jets selection with the requirement $H_{\mathrm{T}}>300 \mathrm{GeV}$ enhances the presence of events with large hadronic activity.

In all phase space regions, we observe a ratio which saturates around $p_{\mathrm{T}} \simeq 300-$ $350 \mathrm{GeV}$. This agrees with the $\mathrm{LO}$ estimations stating that the main distinction between the two processes is the mass difference, with the second difference being the different couplings.

In the inclusive $n_{\text {jets }} \geq 1$ selection (figure 7 ), the plateau value is

$$
R_{\text {dilep }}=\frac{\sigma_{\mathrm{Z} \rightarrow \ell^{+} \ell^{-}}\left(p_{\mathrm{T}}^{\mathrm{Z}}>314 \mathrm{GeV}\right)}{\sigma_{\gamma}\left(p_{\mathrm{T}}^{\gamma}>314 \mathrm{GeV}\right)}=0.0322 \pm 0.0008 \text { (stat) } \pm 0.0020 \text { (syst). }
$$

Here $R_{\text {dilep }}$ is the plateau value of the ratio of the dilepton $\mathrm{Z}$ cross section and the $\gamma+$ jets cross section for the last seven bins $\left(p_{\mathrm{T}}^{V}>314 \mathrm{GeV}\right)$. This translates into the ratio of the total cross sections of $R_{\text {tot }}=0.957 \pm 0.066$ when divided by the average leptonic branching fraction of $(3.3658 \pm 0.0023) \%[51]$.

The estimation from MADGRAPH+PYTHIA6 is overlaid in figures 7 and 8 , where the LO estimation is used to compare $\mathrm{Z}+$ jets and $\gamma+$ jets differential cross sections at the same order of perturbative expansion. Although MADGRAPH+PYTHIA6 does not reproduce the high- $p_{\mathrm{T}}$ tail for either $\mathrm{Z}+$ jets or $\gamma+$ jets, the shapes of the curves are similar for both processes and their ratio is flat. Using LO cross sections, MADGRAPH+PYTHIA6 predicts a ratio with a value of $R_{\mathrm{MG}}=0.0391$, which is higher than that observed in data by a factor of $1.21 \pm 0.08$ (stat+syst). No clear trend away from a flat ratio is observed. Higher-order 

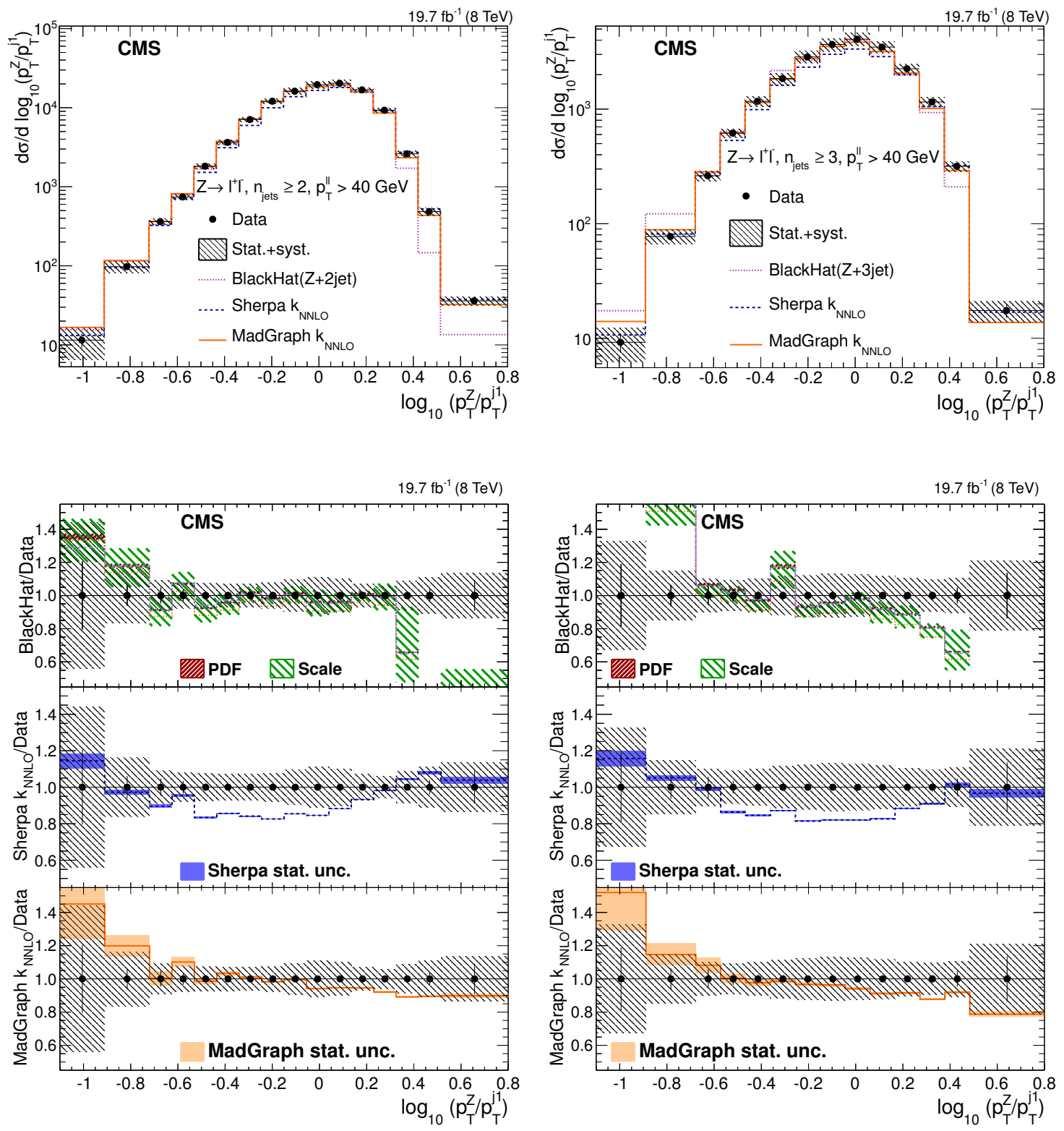

Figure 6. The measured distribution of the observable $\log _{10} p_{\mathrm{T}}^{\mathrm{Z}} / p_{\mathrm{T}}^{\mathrm{j} 1}$ ratio for $n_{\text {jets }} \geq 2$ (top left) and $n_{\text {jets }} \geq 3$ (top right) for $\mathrm{Z}+$ jets in detector-corrected data compared with estimations from MadGraph+Pythia6, Sherpa, and BlackHat. A detailed explanation is given in section 7.1. The bottom plots give the ratio of the various theoretical estimations to the data in the $n_{\text {jets }} \geq 2$ case (bottom left) and $n_{\text {jets }} \geq 3$ case (bottom right). 
effects beyond LO, which could lead to a rise or fall in the plateau region, are smaller than the experimental uncertainties.

The BlaCkHAT estimation is also overlaid in figures 7-8 and reproduces the 1-jet and 2-jet ratio to within $10 \%$ across the entire range. It reproduces the $H_{\mathrm{T}} \geq 300 \mathrm{GeV}$ case accurately in the low- $p_{\mathrm{T}}$ regime and results in an approximately $20 \%$ overestimation in the high $-p_{\mathrm{T}}$ range. In the region where $p_{\mathrm{T}}^{V}<300 \mathrm{GeV}$, the scale uncertainty grows to roughly $30 \%$. This corresponds to the region where BLACKHAT fails to reproduce the $p_{T}^{Z}$ and $p_{\mathrm{T}}^{\gamma}$ spectra separately. Inclusive fixed-order calculations are not designed to model this selection of high jet activity with a comparatively low boson $p_{\mathrm{T}}$. In the 3 -jet case, BlackHAT overestimates the ratio by approximately $25 \%$, but agrees with data starting around the plateau region of approximately $300 \mathrm{GeV}$.

We calculate the scale and PDF uncertainty bands for BLACKHAT using the scale and PDF uncertainty envelopes from the $p_{\mathrm{T}}^{\mathrm{Z}}$ and $p_{\mathrm{T}}^{\gamma}$ spectra. If we correlate the different renormalization and factorization scales $\left(\mu_{R}\right.$ and $\left.\mu_{F}\right)$, the envelope decreases to approximately $2 \%$, whereas if we take the scales as completely anticorrelated, we see a band of approximately $10 \%$ in the bulk. However, we know that the former underestimates the theoretical uncertainty due to renormalization and factorization scales, and the latter overestimates it. The estimation of this uncertainty has been discussed in the literature, and has been examined by comparing different theoretical computational estimations ([9] and [32]). Both of the previously mentioned methods misrepresent the actual uncertainty due to the renormalization and factorization scales. We therefore choose the larger relative scale uncertainty band from each process as an estimate of the uncertainty on the final ratio. Using the NLO cross sections, BLACKHAT predicts the $R_{\text {dilep }}$ ratio with a value of $R_{\mathrm{BH}}=0.03794$, which is higher than that observed in data by a factor of $1.18 \pm 0.14$ (stat + syst).

\section{Summary}

Differential cross sections have been measured for $\mathrm{Z}+$ jets (with $\mathrm{Z} \rightarrow \ell^{+} \ell^{-}$) and isolated $\gamma+$ jets as a function of the boson transverse momentum, using data collected by CMS at $\sqrt{s}=$ $8 \mathrm{TeV}$ corresponding to an integrated luminosity of $19.7 \mathrm{fb}^{-1}$. The estimations from the MC multiparton LO+PS generators MADGRAPH+PYTHIA6 and SHERPA have been compared to the data. We find that the $p_{\mathrm{T}}$ spectra for $\mathrm{Z}+$ jets and $\gamma+$ jets are not well reproduced by these MC models. We observe a monotonic increase of the MC simulation/data ratio with increasing vector boson $p_{\mathrm{T}}$. Using the NLO generator BLACKHAT simulation, we find a smaller discrepancy in shape between data and simulation, indicating that it is likely related to missing higher-order effects.

We have also studied the distribution of the ratios of $p_{\mathrm{T}}^{\mathrm{Z}}$ and hadronic quantities $\left(H_{\mathrm{T}}\right.$ and $\left.p_{\mathrm{T}}^{\mathrm{j} 1}\right)$ in $\mathrm{Z}+$ jets. We find that these agree with the LO+PS estimation over the whole range when an NNLO $K$-factor is applied. The NLO BLACKHAT estimation is accurate in a subrange where the NLO estimation is expected to perform well.

In addition, we presented a measurement of the ratio of the $\mathrm{Z}+$ jets to $\gamma+$ jets cross sections in four phase space regions: $n_{\text {jets }} \geq 1,2,3$, and $H_{\mathrm{T}}>300 \mathrm{GeV}, n_{\text {jets }} \geq 1$. MAdGraph+PYThia6 (LO+PS) overestimates the data by a factor $1.21 \pm 0.08$ (stat+syst), 

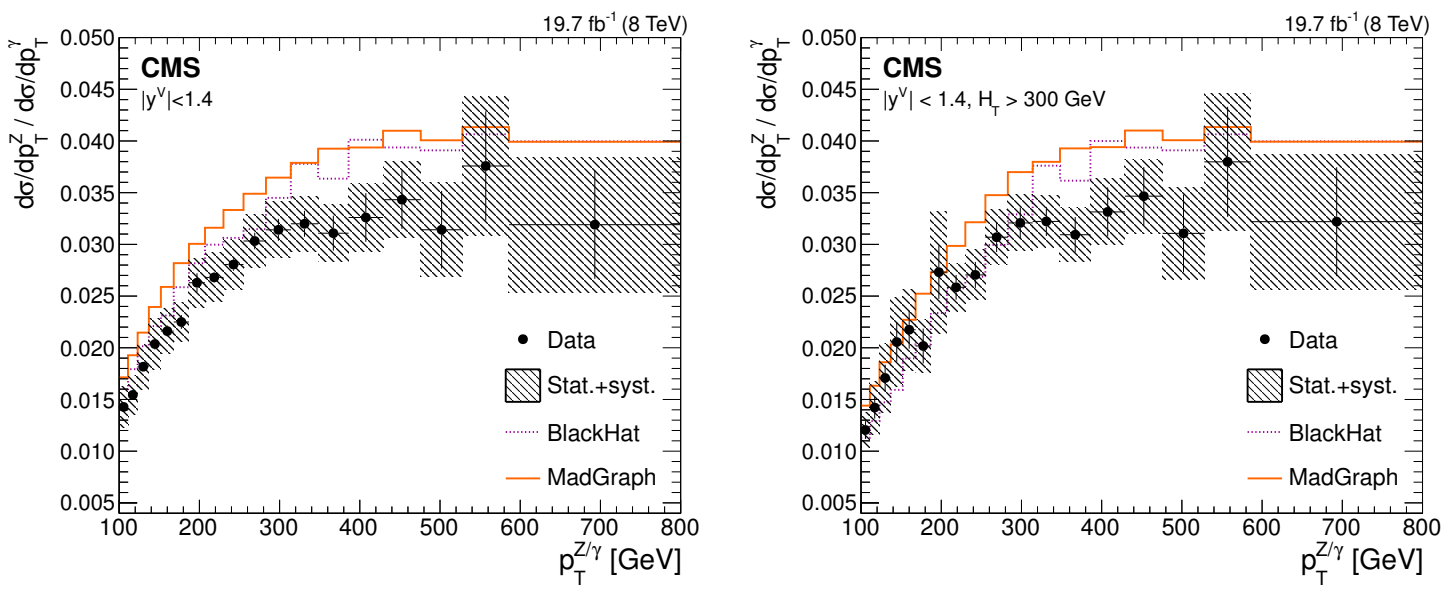

$19.7 \mathrm{fb}^{-1}(8 \mathrm{TeV})$

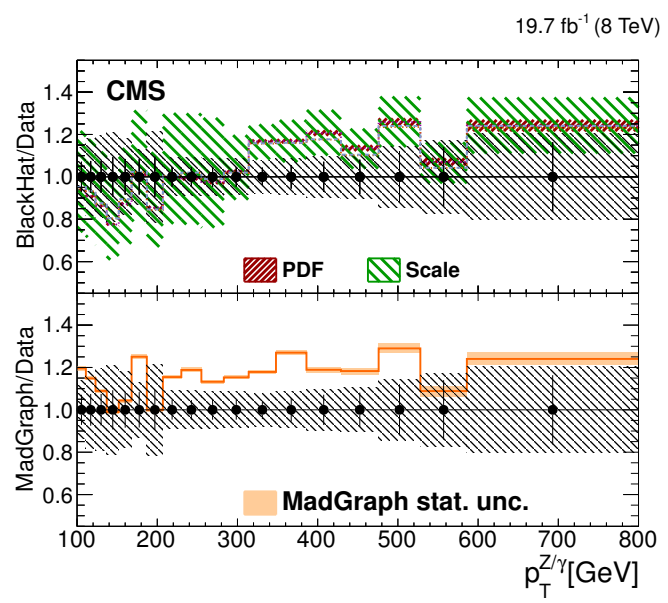

Figure 7. Differential cross section ratio of averaged $\mathrm{Z} \rightarrow\left(\mathrm{e}^{+} \mathrm{e}^{-}+\mu^{+} \mu^{-}\right)$over $\gamma$ as a function of the total transverse-momentum cross section and for central bosons $\left(\left|y^{V}\right|<1.4\right)$ at different kinematic selections in detector-corrected data. Top left: inclusive $\left(n_{\text {jets }} \geq 1\right)$; top right: $H_{\mathrm{T}} \geq$ $300 \mathrm{GeV}, n_{\text {jets }} \geq 1$. The black error bars reflect the statistical uncertainty in the ratio, the hatched (gray) band represents the total uncertainty in the measurement. The shaded band around the MADGRAPH+PYTHIA6 simulation to data ratio represents the statistical uncertainty in the MC estimation. The bottom plots give the ratio of the various theoretical estimations to the data in the $n_{\text {jets }} \geq 1$ case (bottom left) and $H_{\mathrm{T}} \geq 300 \mathrm{GeV}$ case (bottom right).

whereas BlACKHAT (NLO) overestimates the data by a factor $1.18 \pm 0.14$ (stat+syst) in the plateau region i.e., for $p_{\mathrm{T}}^{V}$ above approximately $300 \mathrm{GeV}$. As a function of the vector boson transverse momentum, these factors are at similar values of around 1.2 for all the considered phase space selections. Thus, we find that simulations reproduce the shape of the ratio of $p_{\mathrm{T}}^{\mathrm{Z}}$ to $p_{\mathrm{T}}^{\gamma}$ distributions better than the individual $p_{\mathrm{T}}^{\mathrm{Z}}$ or $p_{\mathrm{T}}^{\gamma}$ distributions in all selections considered. These four selections mimic phase space regions of interest for searches of physics beyond the standard model. We emphasize that the agreement is similar for different jet multiplicities and $H_{\mathrm{T}}$ ranges because $\mathrm{Z}+$ jets and $\gamma+$ jets events have been generated with the same level of accuracy for up to four partons in the final-state ME. 

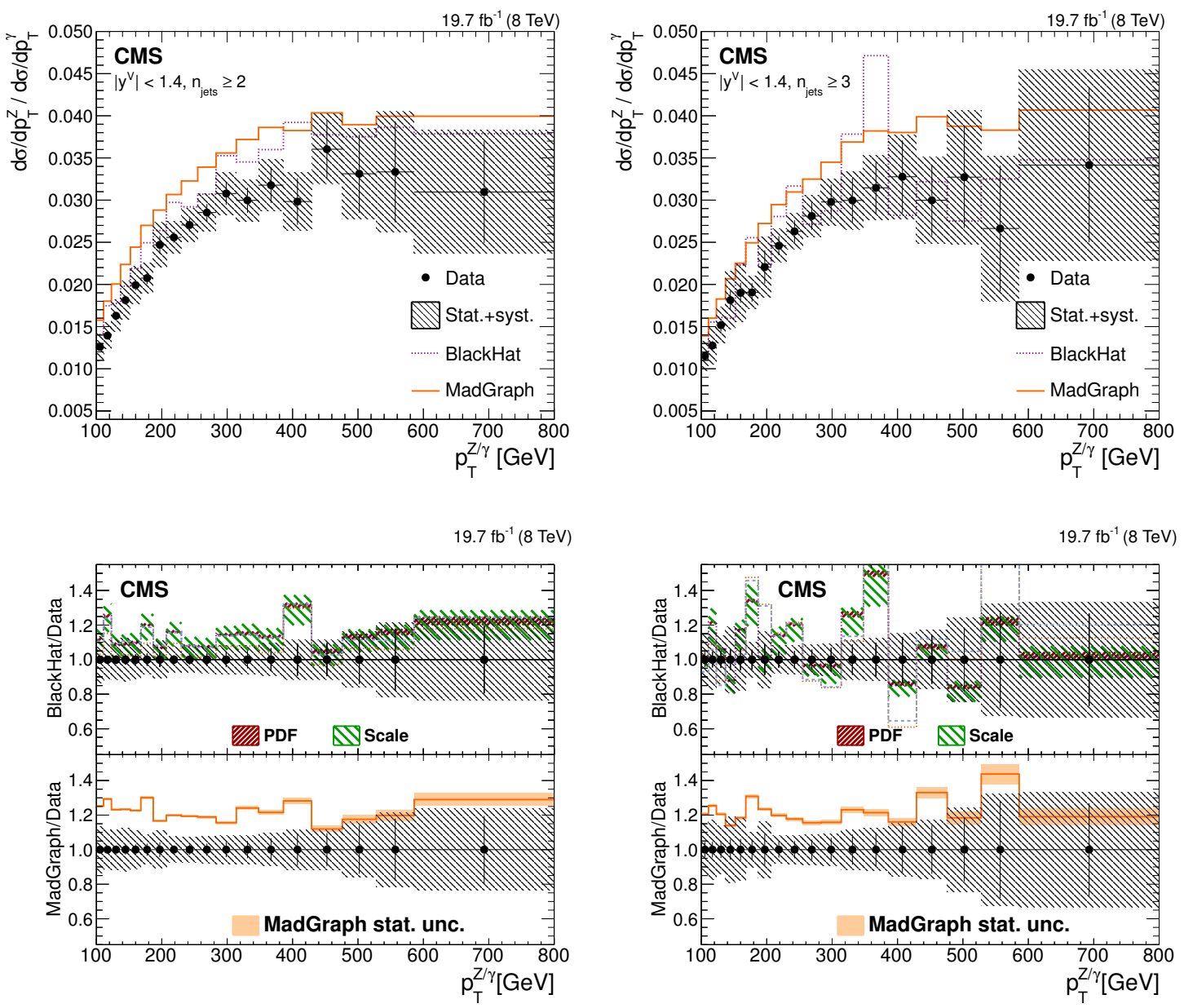

Figure 8. Differential cross section ratio of $\mathrm{Z} \rightarrow\left(\mathrm{e}^{+} \mathrm{e}^{-}+\mu^{+} \mu^{-}\right)$over $\gamma$ as a function of the total transverse-momentum cross section and for central bosons $\left(\left|y^{V}\right|<1.4\right)$ at different kinematic selections in detector-corrected data. Top left: 2 -jet $\left(n_{\text {jets }} \geq 2\right)$; top right: 3 -jet $\left(n_{\text {jets }} \geq 3\right)$. The black error bars reflect the statistical uncertainty in the ratio, the hatched (gray) band represents the total uncertainty in the measurement. The shaded band around the MADGRAPH+PYTHIA6 simulation to data ratio represents the statistical uncertainty in the MC estimation. The bottom plots give the ratio of the various theoretical estimations to the data in the $n_{\text {jets }} \geq 2$ case (bottom left) and $n_{\text {jets }} \geq 3$ case (bottom right).

In the comparison, we considered both processes at either LO or at NLO. It is clear from the differences observed between the NLO and LO+PS estimations in each process, the conclusions may not be true if the samples are generated with different orders of accuracies of the matrix element calculation.

Our results show that properties of the $\mathrm{Z} \rightarrow \nu \bar{\nu}$ process can be predicted using the measured $\gamma+$ jets final state and the simulated ratio between $\mathrm{Z} \rightarrow \nu \bar{\nu}+$ jets and $\gamma+$ jets. However, this simulated ratio must be corrected with the measured ratio of leptonic $\mathrm{Z}+$ jets and $\gamma+$ jets. 


\section{Acknowledgments}

We congratulate our colleagues in the CERN accelerator departments for the excellent performance of the LHC and thank the technical and administrative staffs at CERN and at other CMS institutes for their contributions to the success of the CMS effort. In addition, we gratefully acknowledge the computing centers and personnel of the Worldwide LHC Computing Grid for delivering so effectively the computing infrastructure essential to our analyses. Finally, we acknowledge the enduring support for the construction and operation of the LHC and the CMS detector provided by the following funding agencies: BMWFW and FWF (Austria); FNRS and FWO (Belgium); CNPq, CAPES, FAPERJ, and FAPESP (Brazil); MES (Bulgaria); CERN; CAS, MoST, and NSFC (China); COLCIENCIAS (Colombia); MSES and CSF (Croatia); RPF (Cyprus); MoER, ERC IUT and ERDF (Estonia); Academy of Finland, MEC, and HIP (Finland); CEA and CNRS/IN2P3 (France); BMBF, DFG, and HGF (Germany); GSRT (Greece); OTKA and NIH (Hungary); DAE and DST (India); IPM (Iran); SFI (Ireland); INFN (Italy); MSIP and NRF (Republic of Korea); LAS (Lithuania); MOE and UM (Malaysia); CINVESTAV, CONACYT, SEP, and UASLP-FAI (Mexico); MBIE (New Zealand); PAEC (Pakistan); MSHE and NSC (Poland); FCT (Portugal); JINR (Dubna); MON, RosAtom, RAS and RFBR (Russia); MESTD (Serbia); SEIDI and CPAN (Spain); Swiss Funding Agencies (Switzerland); MST (Taipei); ThEPCenter, IPST, STAR and NSTDA (Thailand); TUBITAK and TAEK (Turkey); NASU and SFFR (Ukraine); STFC (United Kingdom); DOE and NSF (U.S.A.).

Individuals have received support from the Marie-Curie program and the European Research Council and EPLANET (European Union); the Leventis Foundation; the A. P. Sloan Foundation; the Alexander von Humboldt Foundation; the Belgian Federal Science Policy Office; the Fonds pour la Formation à la Recherche dans l'Industrie et dans l'Agriculture (FRIA-Belgium); the Agentschap voor Innovatie door Wetenschap en Technologie (IWTBelgium); the Ministry of Education, Youth and Sports (MEYS) of the Czech Republic; the Council of Science and Industrial Research, India; the HOMING PLUS program of the Foundation for Polish Science, cofinanced from European Union, Regional Development Fund; the Compagnia di San Paolo (Torino); the Consorzio per la Fisica (Trieste); MIUR project 20108T4XTM (Italy); the Thalis and Aristeia programs cofinanced by EU-ESF and the Greek NSRF; and the National Priorities Research Program by Qatar National Research Fund.

Open Access. This article is distributed under the terms of the Creative Commons Attribution License (CC-BY 4.0), which permits any use, distribution and reproduction in any medium, provided the original author(s) and source are credited.

\section{References}

[1] CMS collaboration, Jet production rates in association with $W$ and $Z$ bosons in pp collisions at $\sqrt{s}=7 \mathrm{TeV}$, JHEP 01 (2012) 010 [arXiv:1110.3226] [INSPIRE].

[2] CMS collaboration, Event shapes and azimuthal correlations in $Z+$ jets events in $p p$ collisions at $\sqrt{s}=7 \mathrm{TeV}$, Phys. Lett. B 722 (2013) 238 [arXiv:1301.1646] [inSPIRE]. 
[3] CMS collaboration, Rapidity distributions in exclusive $Z+$ jet and $\gamma+$ jet events in pp collisions at $\sqrt{s}=7$ TeV, Phys. Rev. D 88 (2013) 112009 [arXiv:1310.3082] [InSPIRE].

[4] CMS collaboration, Measurement of the triple-differential cross section for photon+jets production in proton-proton collisions at $\sqrt{s}=7$ TeV, JHEP 06 (2014) 009 [arXiv: 1311.6141] [INSPIRE].

[5] CMS collaboration, Measurements of jet multiplicity and differential production cross sections of $Z+$ jets events in proton-proton collisions at $\sqrt{s}=7$ TeV, Phys. Rev. D 91 (2015) 052008 [arXiv: 1408.3104] [INSPIRE].

[6] ATLAS collaboration, Measurement of the production cross section of jets in association with a $Z$ boson in pp collisions at $\sqrt{s}=7$ TeV with the ATLAS detector, JHEP 07 (2013) 032 [arXiv: 1304.7098] [INSPIRE].

[7] ATLAS collaboration, Measurement of the inclusive isolated prompt photons cross section in pp collisions at $\sqrt{s}=7 \mathrm{TeV}$ with the ATLAS detector using $4.6 \mathrm{fb}^{-1}$, Phys. Rev. D 89 (2014) 052004 [arXiv: 1311.1440] [INSPIRE].

[8] S. Ask, M.A. Parker, T. Sandoval, M.E. Shea and W.J. Stirling, Using $\gamma+$ jets production to calibrate the Standard Model $Z(\rightarrow \nu \bar{\nu})+$ jets background to new physics processes at the LHC, JHEP 10 (2011) 058 [arXiv: 1107.2803] [INSPIRE].

[9] Z. Bern et al., Missing energy and jets for supersymmetry searches, Phys. Rev. D 87 (2013) 034026 [arXiv: 1206.6064] [INSPIRE].

[10] Z. Bern et al., Ntuples for NLO events at hadron colliders, Comput. Phys. Commun. 185 (2014) 1443 [arXiv:1310.7439] [INSPIRE].

[11] CMS collaboration, Searches for supersymmetry using the $M_{T 2}$ variable in hadronic events produced in pp collisions at $8 \mathrm{TeV}$, JHEP 05 (2015) 078 [arXiv: 1502.04358] [INSPIRE].

[12] CMS collaboration, Search for new physics in the multijet and missing transverse momentum final state in proton-proton collisions at $\sqrt{s}=8 \mathrm{TeV}$, JHEP 06 (2014) 055 [arXiv:1402.4770] [INSPIRE].

[13] CMS collaboration, Performance of photon reconstruction and identification with the CMS detector in proton-proton collisions at $\sqrt{s}=8 \mathrm{TeV}, 2015$ JINST $10 \mathrm{P} 08010$ [arXiv: 1502.02702] [INSPIRE].

[14] CMS collaboration, Performance of electron reconstruction and selection with the CMS detector in proton-proton collisions at $\sqrt{s}=8 \mathrm{TeV}, 2015$ JINST $10 \mathrm{P} 06005$ [arXiv: 1502.02701] [INSPIRE].

[15] CMS collaboration, Performance of CMS muon reconstruction in pp collision events at $\sqrt{s}=7$ TeV, 2012 JINST 7 P10002 [arXiv:1206.4071] [INSPIRE].

[16] CMS collaboration, The CMS experiment at the CERN LHC, 2008 JINST 3 S08004 [INSPIRE].

[17] J. Alwall, M. Herquet, F. Maltoni, O. Mattelaer and T. Stelzer, MadGraph 5: going beyond, JHEP 06 (2011) 128 [arXiv: 1106.0522] [INSPIRE].

[18] T. Sjöstrand, S. Mrenna and P.Z. Skands, PYTHIA 6.4 physics and manual, JHEP 05 (2006) 026 [hep-ph/0603175] [INSPIRE].

[19] CMS collaboration, Study of the underlying event at forward rapidity in pp collisions at $\sqrt{s}=0.9,2.76$ and $7 \mathrm{TeV}, \mathrm{JHEP} 04$ (2013) 072 [arXiv: 1302.2394] [INSPIRE]. 
[20] J. Alwall et al., Comparative study of various algorithms for the merging of parton showers and matrix elements in hadronic collisions, Eur. Phys. J. C 53 (2008) 473

[arXiv:0706.2569] [INSPIRE].

[21] J. Pumplin, D.R. Stump, J. Huston, H.L. Lai, P.M. Nadolsky and W.K. Tung, New generation of parton distributions with uncertainties from global QCD analysis, JHEP 07 (2002) 012 [hep-ph/0201195] [INSPIRE].

[22] R. Gavin, Y. Li, F. Petriello and S. Quackenbush, FEWZ 2.0: a code for hadronic Z production at next-to-next-to-leading order, Comput. Phys. Commun. 182 (2011) 2388 [arXiv: 1011.3540] [INSPIRE].

[23] T. Gleisberg et al., Event generation with SHERPA 1.1, JHEP 02 (2009) 007 [arXiv:0811.4622] [INSPIRE].

[24] J. Gao et al., CT10 next-to-next-to-leading order global analysis of QCD, Phys. Rev. D 89 (2014) 033009 [arXiv:1302.6246] [INSPIRE].

[25] M. Czakon, P. Fiedler and A. Mitov, Total top-quark pair-production cross section at hadron colliders through $O\left(\alpha_{S}^{4}\right)$, Phys. Rev. Lett. 110 (2013) 252004 [arXiv:1303.6254] [INSPIRE].

[26] J.M. Campbell and R.K. Ellis, An update on vector boson pair production at hadron colliders, Phys. Rev. D 60 (1999) 113006 [hep-ph/9905386] [INSPIRE].

[27] A.D. Martin, W.J. Stirling, R.S. Thorne and G. Watt, Parton distributions for the LHC, Eur. Phys. J. C 63 (2009) 189 [arXiv:0901.0002] [InSPIRE].

[28] R.D. Ball et al., Parton distributions with LHC data, Nucl. Phys. B 867 (2013) 244 [arXiv:1207.1303] [INSPIRE].

[29] S. Frixione and G. Ridolfi, Jet photoproduction at HERA, Nucl. Phys. B 507 (1997) 315 [hep-ph/9707345] [INSPIRE].

[30] A. Banfi and M. Dasgupta, Dijet rates with symmetric $E_{t}$ cuts, JHEP 01 (2004) 027 [hep-ph/0312108] [INSPIRE].

[31] S. Frixione, Isolated photons in perturbative QCD, Phys. Lett. B 429 (1998) 369 [hep-ph/9801442] [INSPIRE].

[32] Z. Bern et al., Driving missing data at next-to-leading order, Phys. Rev. D 84 (2011) 114002 [arXiv:1106.1423] [INSPIRE].

[33] GEANT4 collaboration, S. Agostinelli et al., GEANT4 - a simulation toolkit, Nucl. Instrum. Meth. A 506 (2003) 250 [inSPIRE].

[34] CMS collaboration, Particle-flow event reconstruction in CMS and performance for jets, taus and MET, CMS-PAS-PFT-09-001, CERN, Geneva Switzerland (2009).

[35] CMS collaboration, Commissioning of the particle-flow event reconstruction with the first LHC collisions recorded in the CMS detector, CMS-PAS-PFT-10-001, CERN, Geneva Switzerland (2010).

[36] M. Cacciari, G.P. Salam and G. Soyez, The catchment area of jets, JHEP 04 (2008) 005 [arXiv:0802.1188] [INSPIRE].

[37] CMS collaboration, Measurement of the inclusive $W$ and $Z$ production cross sections in $p p$ collisions at $\sqrt{s}=7 \mathrm{TeV}$, JHEP 10 (2011) 132 [arXiv:1107.4789] [INSPIRE]. 
[38] W. Adam, R. Fruhwirth, A. Strandlie and T. Todorov, Reconstruction of electrons with the Gaussian sum filter in the CMS tracker at LHC, eConf C 0303241 (2003) TULT009 [physics/0306087] [INSPIRE].

[39] M. Cacciari, G.P. Salam and G. Soyez, The anti- $k_{t}$ jet clustering algorithm, JHEP 04 (2008) 063 [arXiv: 0802.1189] [INSPIRE].

[40] M. Cacciari, G.P. Salam and G. Soyez, FastJet user manual, Eur. Phys. J. C 72 (2012) 1896 [arXiv: 1111.6097] [INSPIRE].

[41] CMS collaboration, Determination of jet energy calibration and transverse momentum resolution in CMS, 2011 JINST 6 P11002 [arXiv:1107.4277] [INSPIRE].

[42] C. Buttar et al., Standard Model handles and candles working group: tools and jets summary report, arXiv:0803.0678 [INSPIRE].

[43] CMS collaboration, Pileup jet identification, CMS-PAS-JME-13-005, CERN, Geneva Switzerland (2013).

[44] G. D'Agostini, A multidimensional unfolding method based on Bayes' theorem, Nucl. Instrum. Meth. A 362 (1995) 487 [INSPIRE].

[45] T. Adye, Unfolding algorithms and tests using RooUnfold, in PHYSTAT 2011 Workshop on Statistical Issues Related to Discovery Claims in Search Experiments and Unfolding, H. Prosper and L. Lyons eds., Geneva Switzerland (2011), pg. 313 [arXiv:1105.1160] [INSPIRE].

[46] L. Lyons, D. Gibaut and P. Clifford, How to combine correlated estimates of a single physical quantity, Nucl. Instrum. Meth. A 270 (1988) 110 [INSPIRE].

[47] CMS collaboration, Measurement of differential cross sections for the production of a pair of isolated photons in pp collisions at $\sqrt{s}=7$ TeV, Eur. Phys. J. C 74 (2014) 3129 [arXiv: 1405.7225] [INSPIRE].

[48] CMS collaboration, CMS luminosity based on pixel cluster counting - Summer 2013 update, CMS-PAS-LUM-13-001, CERN, Geneva Switzerland (2013).

[49] A. Hocker and V. Kartvelishvili, SVD approach to data unfolding, Nucl. Instrum. Meth. A 372 (1996) 469 [hep-ph/9509307] [INSPIRE].

[50] J. Pumplin et al., Uncertainties of predictions from parton distribution functions. 2. The Hessian method, Phys. Rev. D 65 (2001) 014013 [hep-ph/0101032] [INSPIRE].

[51] Particle Data Group collaboration, K.A. Olive et al., Review of particle physics, Chin. Phys. C 38 (2014) 090001 [inSPIRE]. 


\section{The CMS collaboration}

Yerevan Physics Institute, Yerevan, Armenia

V. Khachatryan, A.M. Sirunyan, A. Tumasyan

Institut für Hochenergiephysik der OeAW, Wien, Austria

W. Adam, E. Asilar, T. Bergauer, J. Brandstetter, E. Brondolin, M. Dragicevic, J. Erö, M. Flechl, M. Friedl, R. Frühwirth ${ }^{1}$, V.M. Ghete, C. Hartl, N. Hörmann, J. Hrubec, M. Jeitler ${ }^{1}$, V. Knünz, A. König, M. Krammer ${ }^{1}$, I. Krätschmer, D. Liko, I. Mikulec, D. Rabady ${ }^{2}$, B. Rahbaran, H. Rohringer, J. Schieck ${ }^{1}$, R. Schöfbeck, J. Strauss, W. TrebererTreberspurg, W. Waltenberger, C.-E. Wulz ${ }^{1}$

National Centre for Particle and High Energy Physics, Minsk, Belarus

V. Mossolov, N. Shumeiko, J. Suarez Gonzalez

\section{Universiteit Antwerpen, Antwerpen, Belgium}

S. Alderweireldt, T. Cornelis, E.A. De Wolf, X. Janssen, A. Knutsson, J. Lauwers, S. Luyckx, S. Ochesanu, R. Rougny, M. Van De Klundert, H. Van Haevermaet, P. Van Mechelen, N. Van Remortel, A. Van Spilbeeck

Vrije Universiteit Brussel, Brussel, Belgium

S. Abu Zeid, F. Blekman, J. D'Hondt, N. Daci, I. De Bruyn, K. Deroover, N. Heracleous, J. Keaveney, S. Lowette, L. Moreels, A. Olbrechts, Q. Python, D. Strom, S. Tavernier, W. Van Doninck, P. Van Mulders, G.P. Van Onsem, I. Van Parijs

Université Libre de Bruxelles, Bruxelles, Belgium

P. Barria, C. Caillol, B. Clerbaux, G. De Lentdecker, H. Delannoy, D. Dobur, G. Fasanella, L. Favart, A.P.R. Gay, A. Grebenyuk, A. Léonard, A. Mohammadi, L. Perniè, A. Randleconde, T. Reis, T. Seva, L. Thomas, C. Vander Velde, P. Vanlaer, J. Wang, F. Zenoni

\section{Ghent University, Ghent, Belgium}

K. Beernaert, L. Benucci, A. Cimmino, S. Crucy, A. Fagot, G. Garcia, M. Gul, J. Mccartin, A.A. Ocampo Rios, D. Poyraz, D. Ryckbosch, S. Salva Diblen, M. Sigamani, N. Strobbe, M. Tytgat, W. Van Driessche, E. Yazgan, N. Zaganidis

Université Catholique de Louvain, Louvain-la-Neuve, Belgium

S. Basegmez, C. Beluffi ${ }^{3}$, O. Bondu, G. Bruno, R. Castello, A. Caudron, L. Ceard, G.G. Da Silveira, C. Delaere, T. du Pree, D. Favart, L. Forthomme, A. Giammanco ${ }^{4}$, J. Hollar, A. Jafari, P. Jez, M. Komm, V. Lemaitre, A. Mertens, C. Nuttens, L. Perrini, A. Pin, K. Piotrzkowski, A. Popov ${ }^{5}$, L. Quertenmont, M. Selvaggi, M. Vidal Marono

Université de Mons, Mons, Belgium

N. Beliy, T. Caebergs, G.H. Hammad

Centro Brasileiro de Pesquisas Fisicas, Rio de Janeiro, Brazil

W.L. Aldá Júnior, G.A. Alves, L. Brito, M. Correa Martins Junior, T. Dos Reis Martins, C. Hensel, C. Mora Herrera, A. Moraes, M.E. Pol, P. Rebello Teles 
Universidade do Estado do Rio de Janeiro, Rio de Janeiro, Brazil

E. Belchior Batista Das Chagas, W. Carvalho, J. Chinellato ${ }^{6}$, A. Custódio, E.M. Da Costa, D. De Jesus Damiao, C. De Oliveira Martins, S. Fonseca De Souza, L.M. Huertas Guativa, H. Malbouisson, D. Matos Figueiredo, L. Mundim, H. Nogima, W.L. Prado Da Silva, J. Santaolalla, A. Santoro, A. Sznajder, E.J. Tonelli Manganote ${ }^{6}$, A. Vilela Pereira

Universidade Estadual Paulista $^{a}$, Universidade Federal do ABC ${ }^{b}$, São Paulo, Brazil

S. Ahuja, C.A. Bernardes ${ }^{b}$, S. Dogra ${ }^{a}$, T.R. Fernandez Perez Tomei ${ }^{a}$, E.M. Gregores ${ }^{b}$, P.G. Mercadante ${ }^{b}$, C.S. Moon ${ }^{a, 7}$, S.F. Novaes $^{a}$, Sandra S. Padula ${ }^{a}$, D. Romero Abad, J.C. Ruiz Vargas

Institute for Nuclear Research and Nuclear Energy, Sofia, Bulgaria

A. Aleksandrov, V. Genchev², R. Hadjiiska, P. Iaydjiev, A. Marinov, S. Piperov, M. Rodozov, S. Stoykova, G. Sultanov, M. Vutova

University of Sofia, Sofia, Bulgaria

A. Dimitrov, I. Glushkov, L. Litov, B. Pavlov, P. Petkov

Institute of High Energy Physics, Beijing, China

M. Ahmad, J.G. Bian, G.M. Chen, H.S. Chen, M. Chen, T. Cheng, R. Du, C.H. Jiang, R. Plestina ${ }^{8}$, F. Romeo, S.M. Shaheen, J. Tao, C. Wang, Z. Wang, H. Zhang

State Key Laboratory of Nuclear Physics and Technology, Peking University, Beijing, China

C. Asawatangtrakuldee, Y. Ban, Q. Li, S. Liu, Y. Mao, S.J. Qian, D. Wang, Z. Xu, F. Zhang ${ }^{9}$, L. Zhang, W. Zou

Universidad de Los Andes, Bogota, Colombia

C. Avila, A. Cabrera, L.F. Chaparro Sierra, C. Florez, J.P. Gomez, B. Gomez Moreno, J.C. Sanabria

University of Split, Faculty of Electrical Engineering, Mechanical Engineering and Naval Architecture, Split, Croatia

N. Godinovic, D. Lelas, D. Polic, I. Puljak

University of Split, Faculty of Science, Split, Croatia

Z. Antunovic, M. Kovac

Institute Rudjer Boskovic, Zagreb, Croatia

V. Brigljevic, K. Kadija, J. Luetic, L. Sudic

University of Cyprus, Nicosia, Cyprus

A. Attikis, G. Mavromanolakis, J. Mousa, C. Nicolaou, F. Ptochos, P.A. Razis, H. Rykaczewski

Charles University, Prague, Czech Republic

M. Bodlak, M. Finger, M. Finger Jr. ${ }^{10}$ 
Academy of Scientific Research and Technology of the Arab Republic of Egypt, Egyptian Network of High Energy Physics, Cairo, Egypt

A. Ali ${ }^{11,12}$, R. Aly ${ }^{13}$, S. Aly ${ }^{13}$, Y. Assran ${ }^{14}$, A. Ellithi Kamel ${ }^{15}$, A. Lotfy ${ }^{16}$,

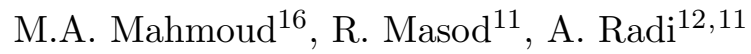

National Institute of Chemical Physics and Biophysics, Tallinn, Estonia

B. Calpas, M. Kadastik, M. Murumaa, M. Raidal, A. Tiko, C. Veelken

Department of Physics, University of Helsinki, Helsinki, Finland

P. Eerola, M. Voutilainen

Helsinki Institute of Physics, Helsinki, Finland

J. Härkönen, V. Karimäki, R. Kinnunen, T. Lampén, K. Lassila-Perini, S. Lehti, T. Lindén,

P. Luukka, T. Mäenpää, T. Peltola, E. Tuominen, J. Tuominiemi, E. Tuovinen, L. Wendland

Lappeenranta University of Technology, Lappeenranta, Finland

J. Talvitie, T. Tuuva

DSM/IRFU, CEA/Saclay, Gif-sur-Yvette, France

M. Besancon, F. Couderc, M. Dejardin, D. Denegri, B. Fabbro, J.L. Faure, C. Favaro, F. Ferri, S. Ganjour, A. Givernaud, P. Gras, G. Hamel de Monchenault, P. Jarry, E. Locci, J. Malcles, J. Rander, A. Rosowsky, M. Titov, A. Zghiche

Laboratoire Leprince-Ringuet, Ecole Polytechnique, IN2P3-CNRS, Palaiseau, France

S. Baffioni, F. Beaudette, P. Busson, L. Cadamuro, E. Chapon, C. Charlot, T. Dahms, O. Davignon, N. Filipovic, A. Florent, R. Granier de Cassagnac, L. Mastrolorenzo, P. Miné, I.N. Naranjo, M. Nguyen, C. Ochando, G. Ortona, P. Paganini, S. Regnard, R. Salerno, J.B. Sauvan, Y. Sirois, T. Strebler, Y. Yilmaz, A. Zabi

Institut Pluridisciplinaire Hubert Curien, Université de Strasbourg, Université de Haute Alsace Mulhouse, CNRS/IN2P3, Strasbourg, France

J.-L. Agram ${ }^{17}$, J. Andrea, A. Aubin, D. Bloch, J.-M. Brom, M. Buttignol, E.C. Chabert, N. Chanon, C. Collard, E. Conte ${ }^{17}$, J.-C. Fontaine ${ }^{17}$, D. Gelé, U. Goerlach, C. Goetzmann, A.-C. Le Bihan, J.A. Merlin², K. Skovpen, P. Van Hove

Centre de Calcul de l'Institut National de Physique Nucleaire et de Physique des Particules, CNRS/IN2P3, Villeurbanne, France

S. Gadrat

Université de Lyon, Université Claude Bernard Lyon 1, CNRS-IN2P3, Institut de Physique Nucléaire de Lyon, Villeurbanne, France

S. Beauceron, N. Beaupere, C. Bernet ${ }^{8}$, G. Boudoul ${ }^{2}$, E. Bouvier, S. Brochet, C.A. Carrillo Montoya, J. Chasserat, R. Chierici, D. Contardo, B. Courbon, P. Depasse, H. El Mamouni, J. Fan, J. Fay, S. Gascon, M. Gouzevitch, B. Ille, I.B. Laktineh, M. Lethuillier, L. Mirabito, A.L. Pequegnot, S. Perries, J.D. Ruiz Alvarez, D. Sabes, L. Sgandurra, V. Sordini, M. Vander Donckt, P. Verdier, S. Viret, H. Xiao 
Institute of High Energy Physics and Informatization, Tbilisi State University, Tbilisi, Georgia

D. Lomidze

\section{RWTH Aachen University, I. Physikalisches Institut, Aachen, Germany}

C. Autermann, S. Beranek, M. Edelhoff, L. Feld, A. Heister, M.K. Kiesel, K. Klein, M. Lipinski, A. Ostapchuk, M. Preuten, F. Raupach, J. Sammet, S. Schael, J.F. Schulte, T. Verlage, H. Weber, B. Wittmer, V. Zhukov ${ }^{5}$

RWTH Aachen University, III. Physikalisches Institut A, Aachen, Germany M. Ata, M. Brodski, E. Dietz-Laursonn, D. Duchardt, M. Endres, M. Erdmann, S. Erdweg, T. Esch, R. Fischer, A. Güth, T. Hebbeker, C. Heidemann, K. Hoepfner, D. Klingebiel, S. Knutzen, P. Kreuzer, M. Merschmeyer, A. Meyer, P. Millet, M. Olschewski, K. Padeken, P. Papacz, T. Pook, M. Radziej, H. Reithler, M. Rieger, L. Sonnenschein, D. Teyssier, S. Thüer

\section{RWTH Aachen University, III. Physikalisches Institut B, Aachen, Germany}

V. Cherepanov, Y. Erdogan, G. Flügge, H. Geenen, M. Geisler, W. Haj Ahmad, F. Hoehle, B. Kargoll, T. Kress, Y. Kuessel, A. Künsken, J. Lingemann ${ }^{2}$, A. Nowack, I.M. Nugent, C. Pistone, O. Pooth, A. Stahl

\section{Deutsches Elektronen-Synchrotron, Hamburg, Germany}

M. Aldaya Martin, I. Asin, N. Bartosik, O. Behnke, U. Behrens, A.J. Bell, K. Borras, A. Burgmeier, A. Cakir, L. Calligaris, A. Campbell, S. Choudhury, F. Costanza, C. Diez Pardos, G. Dolinska, S. Dooling, T. Dorland, G. Eckerlin, D. Eckstein, T. Eichhorn, G. Flucke, E. Gallo, J. Garay Garcia, A. Geiser, A. Gizhko, P. Gunnellini, J. Hauk, M. Hempel ${ }^{18}$, H. Jung, A. Kalogeropoulos, O. Karacheban ${ }^{18}$, M. Kasemann, P. Katsas, J. Kieseler, C. Kleinwort, I. Korol, W. Lange, J. Leonard, K. Lipka, A. Lobanov, R. Mankel, I. Marfin ${ }^{18}$, I.-A. Melzer-Pellmann, A.B. Meyer, G. Mittag, J. Mnich, A. Mussgiller, S. Naumann-Emme, A. Nayak, E. Ntomari, H. Perrey, D. Pitzl, R. Placakyte, A. Raspereza, P.M. Ribeiro Cipriano, B. Roland, M.Ö. Sahin, J. Salfeld-Nebgen, P. Saxena, T. SchoernerSadenius, M. Schröder, C. Seitz, S. Spannagel, K.D. Trippkewitz, C. Wissing

\section{University of Hamburg, Hamburg, Germany}

V. Blobel, M. Centis Vignali, A.R. Draeger, J. Erfle, E. Garutti, K. Goebel, D. Gonzalez, M. Görner, J. Haller, M. Hoffmann, R.S. Höing, A. Junkes, H. Kirschenmann, R. Klanner, R. Kogler, T. Lapsien, T. Lenz, I. Marchesini, D. Marconi, D. Nowatschin, J. Ott, T. Peiffer, A. Perieanu, N. Pietsch, J. Poehlsen, D. Rathjens, C. Sander, H. Schettler, P. Schleper, E. Schlieckau, A. Schmidt, M. Seidel, V. Sola, H. Stadie, G. Steinbrück, H. Tholen, D. Troendle, E. Usai, L. Vanelderen, A. Vanhoefer

\section{Institut für Experimentelle Kernphysik, Karlsruhe, Germany}

M. Akbiyik, C. Barth, C. Baus, J. Berger, C. Böser, E. Butz, T. Chwalek, F. Colombo, W. De Boer, A. Descroix, A. Dierlamm, M. Feindt, F. Frensch, M. Giffels, A. Gilbert, F. Hartmann ${ }^{2}$, U. Husemann, I. Katkov ${ }^{5}$, A. Kornmayer ${ }^{2}$, P. Lobelle Pardo, M.U. Mozer, T. Müller, Th. Müller, M. Plagge, G. Quast, K. Rabbertz, S. Röcker, F. Roscher, H.J. Si- 
monis, F.M. Stober, R. Ulrich, J. Wagner-Kuhr, S. Wayand, T. Weiler, C. Wöhrmann, R. Wolf

Institute of Nuclear and Particle Physics (INPP), NCSR Demokritos, Aghia Paraskevi, Greece

G. Anagnostou, G. Daskalakis, T. Geralis, V.A. Giakoumopoulou, A. Kyriakis, D. Loukas, A. Markou, A. Psallidas, I. Topsis-Giotis

University of Athens, Athens, Greece

A. Agapitos, S. Kesisoglou, A. Panagiotou, N. Saoulidou, E. Tziaferi

University of Ioánnina, Ioánnina, Greece

I. Evangelou, G. Flouris, C. Foudas, P. Kokkas, N. Loukas, N. Manthos, I. Papadopoulos,

E. Paradas, J. Strologas

Wigner Research Centre for Physics, Budapest, Hungary

G. Bencze, C. Hajdu, A. Hazi, P. Hidas, D. Horvath ${ }^{19}$, F. Sikler, V. Veszpremi, G. Vesztergombi ${ }^{20}$, A.J. Zsigmond

Institute of Nuclear Research ATOMKI, Debrecen, Hungary

N. Beni, S. Czellar, J. Karancsi ${ }^{21}$, J. Molnar, J. Palinkas, Z. Szillasi

University of Debrecen, Debrecen, Hungary

M. Bartók ${ }^{22}$, A. Makovec, P. Raics, Z.L. Trocsanyi

National Institute of Science Education and Research, Bhubaneswar, India

P. Mal, K. Mandal, N. Sahoo, S.K. Swain

Panjab University, Chandigarh, India

S. Bansal, S.B. Beri, V. Bhatnagar, R. Chawla, R. Gupta, U.Bhawandeep, A.K. Kalsi, A. Kaur, M. Kaur, R. Kumar, A. Mehta, M. Mittal, N. Nishu, J.B. Singh, G. Walia

University of Delhi, Delhi, India

Ashok Kumar, Arun Kumar, A. Bhardwaj, B.C. Choudhary, R.B. Garg, A. Kumar, S. Malhotra, M. Naimuddin, K. Ranjan, R. Sharma, V. Sharma

Saha Institute of Nuclear Physics, Kolkata, India

S. Banerjee, S. Bhattacharya, K. Chatterjee, S. Dey, S. Dutta, Sa. Jain, Sh. Jain, R. Khurana, N. Majumdar, A. Modak, K. Mondal, S. Mukherjee, S. Mukhopadhyay,

A. Roy, D. Roy, S. Roy Chowdhury, S. Sarkar, M. Sharan

Bhabha Atomic Research Centre, Mumbai, India

A. Abdulsalam, R. Chudasama, D. Dutta, V. Jha, V. Kumar, A.K. Mohanty ${ }^{2}$, L.M. Pant, P. Shukla, A. Topkar

Tata Institute of Fundamental Research, Mumbai, India

T. Aziz, S. Banerjee, S. Bhowmik ${ }^{23}$, R.M. Chatterjee, R.K. Dewanjee, S. Dugad, S. Ganguly, S. Ghosh, M. Guchait, A. Gurtu ${ }^{24}$, G. Kole, S. Kumar, B. Mahakud, M. Maity ${ }^{23}$, G. Majumder, K. Mazumdar, S. Mitra, G.B. Mohanty, B. Parida, T. Sarkar²3, K. Sudhakar, N. Sur, B. Sutar, N. Wickramage ${ }^{25}$ 
Indian Institute of Science Education and Research (IISER), Pune, India

S. Sharma

Institute for Research in Fundamental Sciences (IPM), Tehran, Iran

H. Bakhshiansohi, H. Behnamian, S.M. Etesami ${ }^{26}$, A. Fahim ${ }^{27}$, R. Goldouzian, M. Khakzad, M. Mohammadi Najafabadi, M. Naseri, S. Paktinat Mehdiabadi, F. Rezaei Hosseinabadi, B. Safarzadeh ${ }^{28}$, M. Zeinali

University College Dublin, Dublin, Ireland

M. Felcini, M. Grunewald

INFN Sezione di Bari ${ }^{a}$, Università di Bari ${ }^{b}$, Politecnico di Bari ${ }^{c}$, Bari, Italy M. Abbrescia ${ }^{a, b}$, C. Calabria ${ }^{a, b}$, C. Caputo ${ }^{a, b}$, S.S. Chhibra ${ }^{a, b}$, A. Colaleo $^{a}$, D. Creanza ${ }^{a, c}$, L. Cristella ${ }^{a, b}$, N. De Filippis ${ }^{a, c}$, M. De Palma ${ }^{a, b}$, L. Fiore ${ }^{a}$, G. Iaselli ${ }^{a, c}$, G. Maggi $^{a, c}$, M. Maggi ${ }^{a}$, G. Miniello ${ }^{a, b}$, S. My ${ }^{a, c}$, S. Nuzzo ${ }^{a, b}$, A. Pompili ${ }^{a, b}$, G. Pugliese ${ }^{a, c}$, R. Radogna ${ }^{a, b, 2}$, A. Ranieri ${ }^{a}$, G. Selvaggi ${ }^{a, b}$, A. Sharma ${ }^{a}$, L. Silvestris ${ }^{a, 2}$, R. Venditti ${ }^{a, b}$, P. Verwilligen ${ }^{a}$

INFN Sezione di Bologna ${ }^{a}$, Università di Bologna ${ }^{b}$, Bologna, Italy

G. Abbiendi ${ }^{a}$, C. Battilana, A.C. Benvenuti ${ }^{a}$, D. Bonacorsi ${ }^{a}, b$, S. Braibant-Giacomellia ${ }^{a, b}$,

L. Brigliadori ${ }^{a, b}$, R. Campanini ${ }^{a, b}$, P. Capiluppi ${ }^{a, b}$, A. Castro ${ }^{a, b}$, F.R. Cavallo ${ }^{a}$,

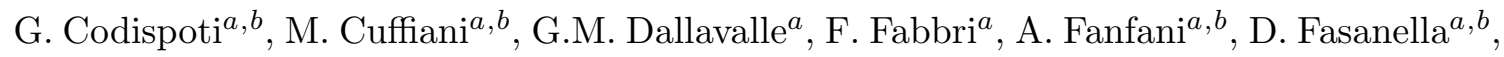
P. Giacomelli ${ }^{a}$, C. Grandi ${ }^{a}$, L. Guiducci ${ }^{a}, b$, S. Marcellini ${ }^{a}$, G. Masetti ${ }^{a}$, A. Montanari ${ }^{a}$,

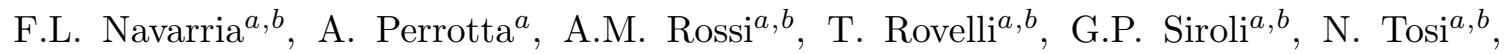
R. Travaglini ${ }^{a, b}$

INFN Sezione di Catania ${ }^{a}$, Università di Catania ${ }^{b}, \operatorname{CSFNSM}^{c}$, Catania, Italy

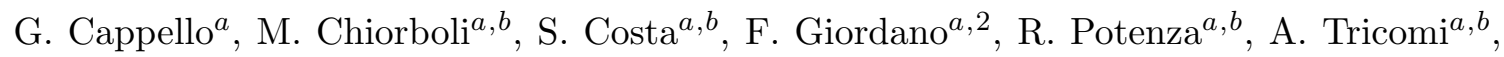
C. Tuve ${ }^{a, b}$

INFN Sezione di Firenze ${ }^{a}$, Università di Firenze ${ }^{b}$, Firenze, Italy

G. Barbagli ${ }^{a}$, V. Ciulli ${ }^{a} b$, C. Civinini ${ }^{a}$, R. D'Alessandro ${ }^{a, b}$, E. Focardi ${ }^{a} b$, S. Gonzi ${ }^{a}, b$, V. Gori ${ }^{a, b}$, P. Lenzi ${ }^{a, b}$, M. Meschini ${ }^{a}$, S. Paoletti ${ }^{a}$, G. Sguazzoni ${ }^{a}$, A. Tropiano ${ }^{a, b}$, L. Viliani ${ }^{a, b}$

INFN Laboratori Nazionali di Frascati, Frascati, Italy

L. Benussi, S. Bianco, F. Fabbri, D. Piccolo

INFN Sezione di Genova ${ }^{a}$, Università di Genova ${ }^{b}$, Genova, Italy

V. Calvelli ${ }^{a, b}$, F. Ferro $^{a}$, M. Lo Vetere ${ }^{a, b}$, E. Robutti $^{a}$, S. Tosi ${ }^{a}, b$

INFN Sezione di Milano-Bicocca ${ }^{a}$, Università di Milano-Bicocca ${ }^{b}$, Milano, Italy

M.E. Dinardo ${ }^{a, b}$, S. Fiorendi ${ }^{a, b}$, S. Gennai ${ }^{a, 2}$, R. Gerosa ${ }^{a, b}$, A. Ghezzi $^{a, b}$, P. Govoni $^{a, b}$, M.T. Lucchini ${ }^{a, b, 2}$, S. Malvezzi ${ }^{a}$, R.A. Manzoni ${ }^{a, b}$, B. Marzocchi ${ }^{a, b, 2}$, D. Menasce ${ }^{a}$, L. Moroni ${ }^{a}$, M. Paganoni ${ }^{a, b}$, D. Pedrini ${ }^{a}$, S. Ragazzi ${ }^{a}, b$, N. Redaelli ${ }^{a}$, T. Tabarelli de Fatis $^{a, b}$ 
INFN Sezione di Napoli ${ }^{a}$, Università di Napoli 'Federico II' ${ }^{b}$, Napoli, Italy, Università della Basilicata ${ }^{c}$, Potenza, Italy, Università G. Marconi ${ }^{d}$, Roma, Italy

S. Buontempo ${ }^{a}$, N. Cavallo $^{a, c}$, S. Di Guida ${ }^{a, d, 2}$, M. Esposito ${ }^{a, b}$, F. Fabozzi ${ }^{a, c}$,

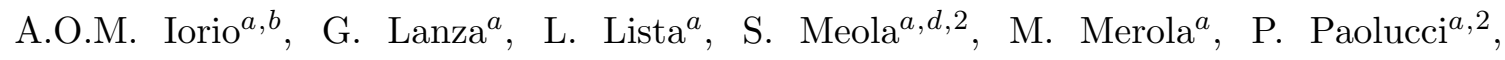
C. Sciacca ${ }^{a, b}$, F. Thyssen

INFN Sezione di Padova ${ }^{a}$, Università di Padova ${ }^{b}$, Padova, Italy, Università di Trento ${ }^{c}$, Trento, Italy

P. Azzi ${ }^{a, 2}$, N. Bacchetta ${ }^{a}$, M. Bellato ${ }^{a}$, D. Bisello ${ }^{a, b}$, R. Carlin ${ }^{a, b}$, A. Carvalho Antunes De Oliveira ${ }^{a, b}$, P. Checchia ${ }^{a}$, M. Dall'Osso ${ }^{a, b}$, T. Dorigo ${ }^{a}$, U. Dosselli ${ }^{a}$, S. Fantinel ${ }^{a}$, F. Gasparini ${ }^{a, b}$, U. Gasparini ${ }^{a, b}$, A. Gozzelino ${ }^{a}$, S. Lacaprara ${ }^{a}$, M. Margoni $^{a}, b$, A.T. Meneguzzo ${ }^{a, b}$, J. Pazzini ${ }^{a, b}$, N. Pozzobon ${ }^{a, b}$, P. Ronchese ${ }^{a, b}$, F. Simonetto ${ }^{a, b}$, E. Torassa ${ }^{a}$, M. Tosi ${ }^{a, b}$, M. Zanetti, P. Zotto ${ }^{a, b}$, A. Zucchetta $^{a, b}$, G. Zumerle ${ }^{a, b}$

INFN Sezione di Pavia ${ }^{a}$, Università di Pavia ${ }^{b}$, Pavia, Italy

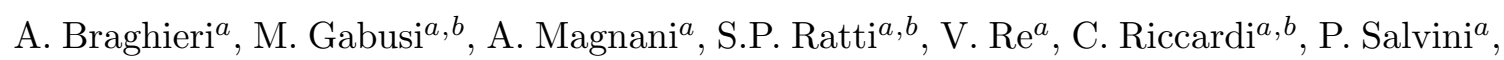
I. $\operatorname{Vai}^{a}$, P. Vitulo ${ }^{a, b}$

INFN Sezione di Perugia ${ }^{a}$, Università di Perugia ${ }^{b}$, Perugia, Italy

L. Alunni Solestizi ${ }^{a, b}$, M. Biasini ${ }^{a, b}$, G.M. Bilei ${ }^{a}$, D. Ciangottini ${ }^{a, b, 2}$, L. Fanò ${ }^{a, b}$, P. Lariccia ${ }^{a, b}$, G. Mantovani ${ }^{a, b}$, M. Menichelli ${ }^{a}$, A. Saha ${ }^{a}$, A. Santocchia ${ }^{a, b}$, A. Spiezia ${ }^{a, b}{ }^{, 2}$

INFN Sezione di Pisa ${ }^{a}$, Università di Pisa ${ }^{b}$, Scuola Normale Superiore di Pisa ${ }^{c}$, Pisa, Italy

K. Androsov ${ }^{a}, 29$, P. Azzurri ${ }^{a}$, G. Bagliesi ${ }^{a}$, J. Bernardini ${ }^{a}$, T. Boccali ${ }^{a}$, G. Broccolo $^{a, c}$, R. Castaldi ${ }^{a}$, M.A. Ciocci ${ }^{a, 29}$, R. Dell'Orso ${ }^{a}$, S. Donato ${ }^{a, c, 2}$, G. Fedi, F. Fiori ${ }^{a, c}$, L. Foà $^{a, c \dagger}{ }^{\text {, }}$ A. Giassi ${ }^{a}$, M.T. Grippo ${ }^{a, 29}$, F. Ligabue ${ }^{a, c}$, T. Lomtadze $^{a}$, L. Martini $^{a, b}$, A. Messineo $^{a, b}$,

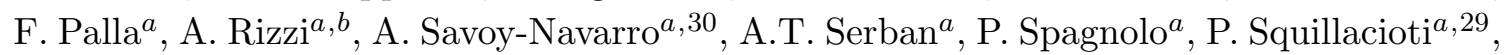
R. Tenchini ${ }^{a}$, G. Tonelli ${ }^{a}, b$, A. Venturi ${ }^{a}$, P.G. Verdini ${ }^{a}$

INFN Sezione di Roma ${ }^{a}$, Università di Roma ${ }^{b}$, Roma, Italy

L. Barone $e^{a, b}$, F. Cavallari ${ }^{a}$, G. D'imperio ${ }^{a, b}$, D. Del Re $e^{a, b}$, M. Diemoz ${ }^{a}$, S. Gelli ${ }^{a, b}$, C. Jorda ${ }^{a}$, E. Longo ${ }^{a, b}$, F. Margaroli ${ }^{a, b}$, P. Meridiani ${ }^{a}$, F. Micheli ${ }^{a, b}$, G. Organtini ${ }^{a, b}$,

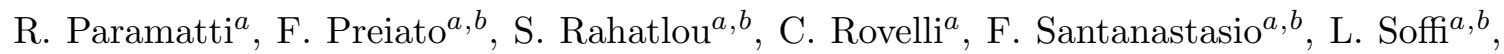
P. Traczyk $k^{a, b, 2}$

INFN Sezione di Torino ${ }^{a}$, Università di Torino ${ }^{b}$, Torino, Italy, Università del Piemonte Orientale ${ }^{c}$, Novara, Italy

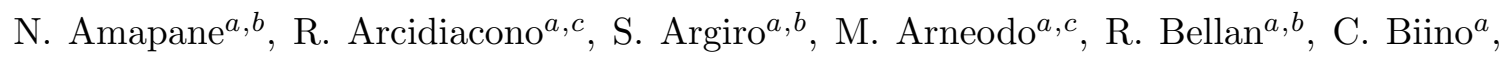
N. Cartiglia ${ }^{a}$, S. Casasso ${ }^{a, b}$, M. Costa ${ }^{a, b}$, R. Covarelli ${ }^{a, b}$, A. Degano ${ }^{a, b}$, N. Demaria ${ }^{a}$, L. Finco ${ }^{a, b, 2}$, B. Kiani ${ }^{a, b}$, C. Mariotti $^{a}$, S. Maselli ${ }^{a}$, E. Migliore ${ }^{a, b}$, V. Monaco ${ }^{a, b}$, M. Musich ${ }^{a}$, M.M. Obertino ${ }^{a, c}$, L. Pacher ${ }^{a, b}$, N. Pastrone ${ }^{a}$, M. Pelliccioni ${ }^{a}$, G.L. Pinna Angioni $^{a, b}$, A. Romero ${ }^{a, b}$, M. Ruspa ${ }^{a, c}$, R. Sacchi ${ }^{a, b}$, A. Solano ${ }^{a, b}$, A. Staiano ${ }^{a}$, U. Tamponi ${ }^{a}$ 
INFN Sezione di Trieste ${ }^{a}$, Università di Trieste ${ }^{b}$, Trieste, Italy

S. Belforte ${ }^{a}$, V. Candelise ${ }^{a, b, 2}$, M. Casarsa ${ }^{a}$, F. Cossutti ${ }^{a}$, G. Della Ricca ${ }^{a, b}$, B. Gobbo ${ }^{a}$,

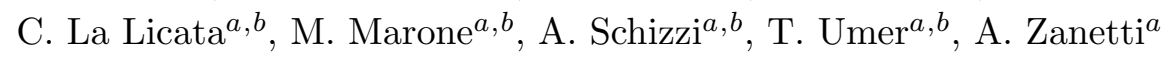

Kangwon National University, Chunchon, Korea

S. Chang, A. Kropivnitskaya, S.K. Nam

Kyungpook National University, Daegu, Korea

D.H. Kim, G.N. Kim, M.S. Kim, D.J. Kong, S. Lee, Y.D. Oh, A. Sakharov, D.C. Son

Chonbuk National University, Jeonju, Korea

H. Kim, T.J. Kim, M.S. Ryu

Chonnam National University, Institute for Universe and Elementary Particles, Kwangju, Korea

S. Song

Korea University, Seoul, Korea

S. Choi, Y. Go, D. Gyun, B. Hong, M. Jo, H. Kim, Y. Kim, B. Lee, K. Lee, K.S. Lee,

S. Lee, S.K. Park, Y. Roh

Seoul National University, Seoul, Korea

H.D. Yoo

University of Seoul, Seoul, Korea

M. Choi, J.H. Kim, J.S.H. Lee, I.C. Park, G. Ryu

Sungkyunkwan University, Suwon, Korea

Y. Choi, Y.K. Choi, J. Goh, D. Kim, E. Kwon, J. Lee, I. Yu

Vilnius University, Vilnius, Lithuania

A. Juodagalvis, J. Vaitkus

National Centre for Particle Physics, Universiti Malaya, Kuala Lumpur, Malaysia

Z.A. Ibrahim, J.R. Komaragiri, M.A.B. Md Ali ${ }^{31}$, F. Mohamad Idris, W.A.T. Wan Abdullah

Centro de Investigacion y de Estudios Avanzados del IPN, Mexico City, Mexico

E. Casimiro Linares, H. Castilla-Valdez, E. De La Cruz-Burelo, I. Heredia-de La Cruz ${ }^{32}$,

A. Hernandez-Almada, R. Lopez-Fernandez, G. Ramirez Sanchez, A. Sanchez-Hernandez

Universidad Iberoamericana, Mexico City, Mexico

S. Carrillo Moreno, F. Vazquez Valencia

Benemerita Universidad Autonoma de Puebla, Puebla, Mexico

S. Carpinteyro, I. Pedraza, H.A. Salazar Ibarguen

Universidad Autónoma de San Luis Potosí, San Luis Potosí, Mexico

A. Morelos Pineda 
University of Auckland, Auckland, New Zealand

D. Krofcheck

University of Canterbury, Christchurch, New Zealand

P.H. Butler, S. Reucroft

National Centre for Physics, Quaid-I-Azam University, Islamabad, Pakistan

A. Ahmad, M. Ahmad, Q. Hassan, H.R. Hoorani, W.A. Khan, T. Khurshid, M. Shoaib

National Centre for Nuclear Research, Swierk, Poland

H. Bialkowska, M. Bluj, B. Boimska, T. Frueboes, M. Górski, M. Kazana, K. Nawrocki,

K. Romanowska-Rybinska, M. Szleper, P. Zalewski

Institute of Experimental Physics, Faculty of Physics, University of Warsaw, Warsaw, Poland

G. Brona, K. Bunkowski, K. Doroba, A. Kalinowski, M. Konecki, J. Krolikowski, M. Misiura, M. Olszewski, M. Walczak

Laboratório de Instrumentação e Física Experimental de Partículas, Lisboa, Portugal

P. Bargassa, C. Beirão Da Cruz E Silva, A. Di Francesco, P. Faccioli, P.G. Ferreira Parracho,

M. Gallinaro, L. Lloret Iglesias, F. Nguyen, J. Rodrigues Antunes, J. Seixas, O. Toldaiev,

D. Vadruccio, J. Varela, P. Vischia

Joint Institute for Nuclear Research, Dubna, Russia

S. Afanasiev, P. Bunin, M. Gavrilenko, I. Golutvin, I. Gorbunov, A. Kamenev, V. Karjavin, V. Konoplyanikov, A. Lanev, A. Malakhov, V. Matveev ${ }^{33}$, P. Moisenz, V. Palichik, V. Perelygin, S. Shmatov, S. Shulha, N. Skatchkov, V. Smirnov, T. Toriashvili ${ }^{34}$, A. Zarubin

Petersburg Nuclear Physics Institute, Gatchina (St. Petersburg), Russia

V. Golovtsov, Y. Ivanov, V. Kim5 ${ }^{35}$ E. Kuznetsova, P. Levchenko, V. Murzin, V. Oreshkin, I. Smirnov, V. Sulimov, L. Uvarov, S. Vavilov, A. Vorobyev

Institute for Nuclear Research, Moscow, Russia

Yu. Andreev, A. Dermenev, S. Gninenko, N. Golubev, A. Karneyeu, M. Kirsanov, N. Krasnikov, A. Pashenkov, D. Tlisov, A. Toropin

Institute for Theoretical and Experimental Physics, Moscow, Russia

V. Epshteyn, V. Gavrilov, N. Lychkovskaya, V. Popov, I. Pozdnyakov, G. Safronov, A. Spiridonov, E. Vlasov, A. Zhokin

National Research Nuclear University 'Moscow Engineering Physics Institute' (MEPhI), Moscow, Russia

A. Bylinkin

P.N. Lebedev Physical Institute, Moscow, Russia

V. Andreev, M. Azarkin ${ }^{36}$, I. Dremin ${ }^{36}$, M. Kirakosyan, A. Leonidov ${ }^{36}$, G. Mesyats, S.V. Rusakov, A. Vinogradov 
Skobeltsyn Institute of Nuclear Physics, Lomonosov Moscow State University, Moscow, Russia

A. Baskakov, A. Belyaev, E. Boos, M. Dubinin ${ }^{37}$, L. Dudko, A. Ershov, A. Gribushin, V. Klyukhin, O. Kodolova, I. Lokhtin, I. Myagkov, S. Obraztsov, S. Petrushanko, V. Savrin, A. Snigirev

State Research Center of Russian Federation, Institute for High Energy Physics, Protvino, Russia

I. Azhgirey, I. Bayshev, S. Bitioukov, V. Kachanov, A. Kalinin, D. Konstantinov, V. Krychkine, V. Petrov, R. Ryutin, A. Sobol, L. Tourtchanovitch, S. Troshin, N. Tyurin, A. Uzunian, A. Volkov

University of Belgrade, Faculty of Physics and Vinca Institute of Nuclear Sciences, Belgrade, Serbia

P. Adzic ${ }^{38}$, M. Ekmedzic, J. Milosevic, V. Rekovic

Centro de Investigaciones Energéticas Medioambientales y Tecnológicas (CIEMAT), Madrid, Spain

J. Alcaraz Maestre, E. Calvo, M. Cerrada, M. Chamizo Llatas, N. Colino, B. De La Cruz, A. Delgado Peris, D. Domínguez Vázquez, A. Escalante Del Valle, C. Fernandez Bedoya, J.P. Fernández Ramos, J. Flix, M.C. Fouz, P. Garcia-Abia, O. Gonzalez Lopez, S. Goy Lopez, J.M. Hernandez, M.I. Josa, E. Navarro De Martino, A. Pérez-Calero Yzquierdo, J. Puerta Pelayo, A. Quintario Olmeda, I. Redondo, L. Romero, M.S. Soares

Universidad Autónoma de Madrid, Madrid, Spain

C. Albajar, J.F. de Trocóniz, M. Missiroli, D. Moran

Universidad de Oviedo, Oviedo, Spain

H. Brun, J. Cuevas, J. Fernandez Menendez, S. Folgueras, I. Gonzalez Caballero, E. Palencia Cortezon, J.M. Vizan Garcia

Instituto de Física de Cantabria (IFCA), CSIC-Universidad de Cantabria, Santander, Spain

J.A. Brochero Cifuentes, I.J. Cabrillo, A. Calderon, J.R. Castiñeiras De Saa, J. Duarte Campderros, M. Fernandez, G. Gomez, A. Graziano, A. Lopez Virto, J. Marco, R. Marco,

C. Martinez Rivero, F. Matorras, F.J. Munoz Sanchez, J. Piedra Gomez, T. Rodrigo, A.Y. Rodríguez-Marrero, A. Ruiz-Jimeno, L. Scodellaro, I. Vila, R. Vilar Cortabitarte

CERN, European Organization for Nuclear Research, Geneva, Switzerland

D. Abbaneo, E. Auffray, G. Auzinger, M. Bachtis, P. Baillon, A.H. Ball, D. Barney, A. Benaglia, J. Bendavid, L. Benhabib, J.F. Benitez, G.M. Berruti, G. Bianchi, P. Bloch, A. Bocci, A. Bonato, C. Botta, H. Breuker, T. Camporesi, G. Cerminara, S. Colafranceschi ${ }^{39}$, M. D'Alfonso, D. d'Enterria, A. Dabrowski, V. Daponte, A. David, M. De Gruttola, F. De Guio, A. De Roeck, S. De Visscher, E. Di Marco, M. Dobson, M. Dordevic, N. Dupont-Sagorin, A. Elliott-Peisert, J. Eugster, G. Franzoni, W. Funk, D. Gigi, K. Gill, D. Giordano, M. Girone, F. Glege, R. Guida, S. Gundacker, M. Guthoff, J. Hammer, M. Hansen, P. Harris, J. Hegeman, V. Innocente, P. Janot, M.J. Kortelainen, 
K. Kousouris, K. Krajczar, P. Lecoq, C. Lourenço, N. Magini, L. Malgeri, M. Mannelli, J. Marrouche, A. Martelli, L. Masetti, F. Meijers, S. Mersi, E. Meschi, F. Moortgat, S. Morovic, M. Mulders, M.V. Nemallapudi, H. Neugebauer, S. Orfanelli, L. Orsini, L. Pape, E. Perez, A. Petrilli, G. Petrucciani, A. Pfeiffer, D. Piparo, A. Racz, G. Rolandi ${ }^{40}$, M. Rovere, M. Ruan, H. Sakulin, C. Schäfer, C. Schwick, A. Sharma, P. Silva, M. Simon, P. Sphicas ${ }^{41}$, D. Spiga, J. Steggemann, B. Stieger, M. Stoye, Y. Takahashi, D. Treille, A. Tsirou, G.I. $\operatorname{Veres}^{20}$, N. Wardle, H.K. Wöhri, A. Zagozdzinska ${ }^{42}$, W.D. Zeuner

\section{Paul Scherrer Institut, Villigen, Switzerland}

W. Bertl, K. Deiters, W. Erdmann, R. Horisberger, Q. Ingram, H.C. Kaestli, D. Kotlinski, U. Langenegger, T. Rohe

Institute for Particle Physics, ETH Zurich, Zurich, Switzerland

F. Bachmair, L. Bäni, L. Bianchini, M.A. Buchmann, B. Casal, G. Dissertori, M. Dittmar, M. Donegà, M. Dünser, P. Eller, C. Grab, C. Heidegger, D. Hits, J. Hoss, G. Kasieczka, W. Lustermann, B. Mangano, A.C. Marini, M. Marionneau, P. Martinez Ruiz del Arbol, M. Masciovecchio, D. Meister, N. Mohr, P. Musella, F. Nessi-Tedaldi, F. Pandolfi, J. Pata, F. Pauss, L. Perrozzi, M. Peruzzi, M. Quittnat, M. Rossini, A. Starodumov ${ }^{43}$, M. Takahashi, V.R. Tavolaro, K. Theofilatos, R. Wallny, H.A. Weber

\section{Universität Zürich, Zurich, Switzerland}

T.K. Aarrestad, C. Amsler ${ }^{4}$, M.F. Canelli, V. Chiochia, A. De Cosa, C. Galloni, A. Hinzmann, T. Hreus, B. Kilminster, C. Lange, J. Ngadiuba, D. Pinna, P. Robmann, F.J. Ronga, D. Salerno, S. Taroni, Y. Yang

\section{National Central University, Chung-Li, Taiwan}

M. Cardaci, K.H. Chen, T.H. Doan, C. Ferro, M. Konyushikhin, C.M. Kuo, W. Lin, Y.J. Lu, R. Volpe, S.S. Yu

\section{National Taiwan University (NTU), Taipei, Taiwan}

P. Chang, Y.H. Chang, Y.W. Chang, Y. Chao, K.F. Chen, P.H. Chen, C. Dietz,

U. Grundler, W.-S. Hou, Y. Hsiung, Y.F. Liu, R.-S. Lu, M. Miñano Moya, E. Petrakou, J.f. Tsai, Y.M. Tzeng, R. Wilken

Chulalongkorn University, Faculty of Science, Department of Physics, Bangkok, Thailand

B. Asavapibhop, K. Kovitanggoon, G. Singh, N. Srimanobhas, N. Suwonjandee

\section{Cukurova University, Adana, Turkey}

A. Adiguzel, M.N. Bakirci ${ }^{45}$, C. Dozen, I. Dumanoglu, E. Eskut, S. Girgis, G. Gokbulut, Y. Guler, E. Gurpinar, I. Hos, E.E. Kangal ${ }^{46}$, G. Onengut ${ }^{47}$, K. Ozdemir ${ }^{48}$, A. Polatoz, D. Sunar $\mathrm{Cerci}^{49}$, M. Vergili, C. Zorbilmez

Middle East Technical University, Physics Department, Ankara, Turkey I.V. Akin, B. Bilin, S. Bilmis, B. Isildak ${ }^{50}$, G. Karapinar ${ }^{51}$, U.E. Surat, M. Yalvac, M. Zeyrek 
Bogazici University, Istanbul, Turkey

E.A. Albayrak ${ }^{52}$, E. Gülmez, M. Kaya ${ }^{53}$, O. Kaya ${ }^{54}$, T. Yetkin ${ }^{55}$

Istanbul Technical University, Istanbul, Turkey

K. Cankocak, Y.O. Günaydin ${ }^{56}$, F.I. Vardarlı

Institute for Scintillation Materials of National Academy of Science of Ukraine, Kharkov, Ukraine

B. Grynyov

National Scientific Center, Kharkov Institute of Physics and Technology, Kharkov, Ukraine

L. Levchuk, P. Sorokin

University of Bristol, Bristol, United Kingdom

R. Aggleton, F. Ball, L. Beck, J.J. Brooke, E. Clement, D. Cussans, H. Flacher, J. Goldstein, M. Grimes, G.P. Heath, H.F. Heath, J. Jacob, L. Kreczko, C. Lucas, Z. Meng, D.M. Newbold ${ }^{57}$, S. Paramesvaran, A. Poll, T. Sakuma, S. Seif El Nasr-storey, S. Senkin, D. Smith, V.J. Smith

Rutherford Appleton Laboratory, Didcot, United Kingdom

K.W. Bell, A. Belyaev ${ }^{58}$, C. Brew, R.M. Brown, D.J.A. Cockerill, J.A. Coughlan, K. Harder, S. Harper, E. Olaiya, D. Petyt, C.H. Shepherd-Themistocleous, A. Thea, I.R. Tomalin, T. Williams, W.J. Womersley, S.D. Worm

\section{Imperial College, London, United Kingdom}

M. Baber, R. Bainbridge, O. Buchmuller, A. Bundock, D. Burton, M. Citron, D. Colling, L. Corpe, N. Cripps, P. Dauncey, G. Davies, A. De Wit, M. Della Negra, P. Dunne, A. Elwood, W. Ferguson, J. Fulcher, D. Futyan, G. Hall, G. Iles, G. Karapostoli, M. Kenzie, R. Lane, R. Lucas ${ }^{57}$, L. Lyons, A.-M. Magnan, S. Malik, J. Nash, A. Nikitenko ${ }^{43}$, J. Pela, M. Pesaresi, K. Petridis, D.M. Raymond, A. Richards, A. Rose, C. Seez, P. Sharp ${ }^{\dagger}$, A. Tapper, K. Uchida, M. Vazquez Acosta, T. Virdee, S.C. Zenz

Brunel University, Uxbridge, United Kingdom

J.E. Cole, P.R. Hobson, A. Khan, P. Kyberd, D. Leggat, D. Leslie, I.D. Reid, P. Symonds, L. Teodorescu, M. Turner

Baylor University, Waco, U.S.A.

J. Dittmann, K. Hatakeyama, A. Kasmi, H. Liu, N. Pastika, T. Scarborough

The University of Alabama, Tuscaloosa, U.S.A.

O. Charaf, S.I. Cooper, C. Henderson, P. Rumerio

Boston University, Boston, U.S.A.

A. Avetisyan, T. Bose, C. Fantasia, D. Gastler, P. Lawson, D. Rankin, C. Richardson, J. Rohlf, J. St. John, L. Sulak, D. Zou 
Brown University, Providence, U.S.A.

J. Alimena, E. Berry, S. Bhattacharya, D. Cutts, Z. Demiragli, N. Dhingra, A. Ferapontov, A. Garabedian, U. Heintz, E. Laird, G. Landsberg, Z. Mao, M. Narain, S. Sagir, T. Sinthuprasith

University of California, Davis, Davis, U.S.A.

R. Breedon, G. Breto, M. Calderon De La Barca Sanchez, S. Chauhan, M. Chertok, J. Conway, R. Conway, P.T. Cox, R. Erbacher, M. Gardner, W. Ko, R. Lander, M. Mulhearn, D. Pellett, J. Pilot, F. Ricci-Tam, S. Shalhout, J. Smith, M. Squires, D. Stolp, M. Tripathi, S. Wilbur, R. Yohay

University of California, Los Angeles, U.S.A.

R. Cousins, P. Everaerts, C. Farrell, J. Hauser, M. Ignatenko, G. Rakness, D. Saltzberg,

E. Takasugi, V. Valuev, M. Weber

University of California, Riverside, Riverside, U.S.A.

K. Burt, R. Clare, J. Ellison, J.W. Gary, G. Hanson, J. Heilman, M. Ivova Rikova, P. Jandir, E. Kennedy, F. Lacroix, O.R. Long, A. Luthra, M. Malberti, M. Olmedo Negrete, A. Shrinivas, S. Sumowidagdo, H. Wei, S. Wimpenny

University of California, San Diego, La Jolla, U.S.A.

J.G. Branson, G.B. Cerati, S. Cittolin, R.T. D'Agnolo, A. Holzner, R. Kelley, D. Klein, D. Kovalskyi, J. Letts, I. Macneill, D. Olivito, S. Padhi, M. Pieri, M. Sani, V. Sharma, S. Simon, M. Tadel, Y. Tu, A. Vartak, S. Wasserbaech ${ }^{59}$, C. Welke, F. Würthwein, A. Yagil, G. Zevi Della Porta

University of California, Santa Barbara, Santa Barbara, U.S.A.

D. Barge, J. Bradmiller-Feld, C. Campagnari, A. Dishaw, V. Dutta, K. Flowers, M. Franco Sevilla, P. Geffert, C. George, F. Golf, L. Gouskos, J. Gran, J. Incandela, C. Justus, N. Mccoll, S.D. Mullin, J. Richman, D. Stuart, W. To, C. West, J. Yoo

California Institute of Technology, Pasadena, U.S.A.

D. Anderson, A. Apresyan, A. Bornheim, J. Bunn, Y. Chen, J. Duarte, A. Mott, H.B. Newman, C. Pena, M. Pierini, M. Spiropulu, J.R. Vlimant, S. Xie, R.Y. Zhu

Carnegie Mellon University, Pittsburgh, U.S.A.

V. Azzolini, A. Calamba, B. Carlson, T. Ferguson, Y. Iiyama, M. Paulini, J. Russ, M. Sun, H. Vogel, I. Vorobiev

University of Colorado at Boulder, Boulder, U.S.A.

J.P. Cumalat, W.T. Ford, A. Gaz, F. Jensen, A. Johnson, M. Krohn, T. Mulholland, U. Nauenberg, J.G. Smith, K. Stenson, S.R. Wagner

Cornell University, Ithaca, U.S.A.

J. Alexander, A. Chatterjee, J. Chaves, J. Chu, S. Dittmer, N. Eggert, N. Mirman, G. Nicolas Kaufman, J.R. Patterson, A. Ryd, L. Skinnari, W. Sun, S.M. Tan, W.D. Teo, J. Thom, J. Thompson, J. Tucker, Y. Weng, P. Wittich 
Fermi National Accelerator Laboratory, Batavia, U.S.A.

S. Abdullin, M. Albrow, J. Anderson, G. Apollinari, L.A.T. Bauerdick, A. Beretvas, J. Berryhill, P.C. Bhat, G. Bolla, K. Burkett, J.N. Butler, H.W.K. Cheung, F. Chlebana, S. Cihangir, V.D. Elvira, I. Fisk, J. Freeman, E. Gottschalk, L. Gray, D. Green, S. Grünendahl, O. Gutsche, J. Hanlon, D. Hare, R.M. Harris, J. Hirschauer, B. Hooberman, Z. Hu, S. Jindariani, M. Johnson, U. Joshi, A.W. Jung, B. Klima, B. Kreis, S. Kwan ${ }^{\dagger}$, S. Lammel, J. Linacre, D. Lincoln, R. Lipton, T. Liu, R. Lopes De Sá, J. Lykken, K. Maeshima, J.M. Marraffino, V.I. Martinez Outschoorn, S. Maruyama, D. Mason, P. McBride, P. Merkel, K. Mishra, S. Mrenna, S. Nahn, C. Newman-Holmes, V. O'Dell, O. Prokofyev, E. Sexton-Kennedy, A. Soha, W.J. Spalding, L. Spiegel, L. Taylor, S. Tkaczyk, N.V. Tran, L. Uplegger, E.W. Vaandering, C. Vernieri, M. Verzocchi, R. Vidal, A. Whitbeck, F. Yang, H. Yin

University of Florida, Gainesville, U.S.A.

D. Acosta, P. Avery, P. Bortignon, D. Bourilkov, A. Carnes, M. Carver, D. Curry, S. Das, G.P. Di Giovanni, R.D. Field, M. Fisher, I.K. Furic, J. Hugon, J. Konigsberg, A. Korytov, T. Kypreos, J.F. Low, P. Ma, K. Matchev, H. Mei, P. Milenovic ${ }^{60}$, G. Mitselmakher, L. Muniz, D. Rank, A. Rinkevicius, L. Shchutska, M. Snowball, D. Sperka, S.J. Wang, J. Yelton

Florida International University, Miami, U.S.A.

S. Hewamanage, S. Linn, P. Markowitz, G. Martinez, J.L. Rodriguez

Florida State University, Tallahassee, U.S.A.

A. Ackert, J.R. Adams, T. Adams, A. Askew, J. Bochenek, B. Diamond, J. Haas, S. Hagopian, V. Hagopian, K.F. Johnson, A. Khatiwada, H. Prosper, V. Veeraraghavan, M. Weinberg

Florida Institute of Technology, Melbourne, U.S.A.

V. Bhopatkar, M. Hohlmann, H. Kalakhety, D. Mareskas-palcek, T. Roy, F. Yumiceva University of Illinois at Chicago (UIC), Chicago, U.S.A.

M.R. Adams, L. Apanasevich, D. Berry, R.R. Betts, I. Bucinskaite, R. Cavanaugh, O. Evdokimov, L. Gauthier, C.E. Gerber, D.J. Hofman, P. Kurt, C. O'Brien, I.D. Sandoval Gonzalez, C. Silkworth, P. Turner, N. Varelas, Z. Wu, M. Zakaria

The University of Iowa, Iowa City, U.S.A.

B. Bilki ${ }^{61}$, W. Clarida, K. Dilsiz, R.P. Gandrajula, M. Haytmyradov, V. Khristenko, J.P. Merlo, H. Mermerkaya ${ }^{62}$, A. Mestvirishvili, A. Moeller, J. Nachtman, H. Ogul, Y. Onel, F. $\mathrm{Ozok}^{52}$, A. Penzo, S. Sen, C. Snyder, P. Tan, E. Tiras, J. Wetzel, K. Yi

Johns Hopkins University, Baltimore, U.S.A.

I. Anderson, B.A. Barnett, B. Blumenfeld, D. Fehling, L. Feng, A.V. Gritsan, P. Maksimovic, C. Martin, K. Nash, M. Osherson, M. Swartz, M. Xiao, Y. Xin

The University of Kansas, Lawrence, U.S.A.

P. Baringer, A. Bean, G. Benelli, C. Bruner, J. Gray, R.P. Kenny III, D. Majumder, M. Malek, M. Murray, D. Noonan, S. Sanders, R. Stringer, Q. Wang, J.S. Wood 
Kansas State University, Manhattan, U.S.A.

I. Chakaberia, A. Ivanov, K. Kaadze, S. Khalil, M. Makouski, Y. Maravin, L.K. Saini, N. Skhirtladze, I. Svintradze

Lawrence Livermore National Laboratory, Livermore, U.S.A.

D. Lange, F. Rebassoo, D. Wright

University of Maryland, College Park, U.S.A.

C. Anelli, A. Baden, O. Baron, A. Belloni, B. Calvert, S.C. Eno, C. Ferraioli, J.A. Gomez, N.J. Hadley, S. Jabeen, R.G. Kellogg, T. Kolberg, J. Kunkle, Y. Lu, A.C. Mignerey, K. Pedro, Y.H. Shin, A. Skuja, M.B. Tonjes, S.C. Tonwar

Massachusetts Institute of Technology, Cambridge, U.S.A.

A. Apyan, R. Barbieri, A. Baty, K. Bierwagen, S. Brandt, W. Busza, I.A. Cali, L. Di Matteo, G. Gomez Ceballos, M. Goncharov, D. Gulhan, M. Klute, Y.S. Lai, Y.-J. Lee, A. Levin, P.D. Luckey, C. Mcginn, X. Niu, C. Paus, D. Ralph, C. Roland, G. Roland, G.S.F. Stephans, K. Sumorok, M. Varma, D. Velicanu, J. Veverka, J. Wang, T.W. Wang, B. Wyslouch, M. Yang, V. Zhukova

University of Minnesota, Minneapolis, U.S.A.

B. Dahmes, A. Finkel, A. Gude, S.C. Kao, K. Klapoetke, Y. Kubota, J. Mans, S. Nourbakhsh, R. Rusack, N. Tambe, J. Turkewitz

University of Mississippi, Oxford, U.S.A.

J.G. Acosta, S. Oliveros

University of Nebraska-Lincoln, Lincoln, U.S.A.

E. Avdeeva, K. Bloom, S. Bose, D.R. Claes, A. Dominguez, C. Fangmeier, R. Gonzalez Suarez, R. Kamalieddin, J. Keller, D. Knowlton, I. Kravchenko, J. Lazo-Flores, F. Meier, J. Monroy, F. Ratnikov, J.E. Siado, G.R. Snow

State University of New York at Buffalo, Buffalo, U.S.A.

M. Alyari, J. Dolen, J. George, A. Godshalk, I. Iashvili, J. Kaisen, A. Kharchilava, A. Kumar, S. Rappoccio

\section{Northeastern University, Boston, U.S.A.}

G. Alverson, E. Barberis, D. Baumgartel, M. Chasco, A. Hortiangtham, A. Massironi, D.M. Morse, D. Nash, T. Orimoto, R. Teixeira De Lima, D. Trocino, R.-J. Wang, D. Wood, J. Zhang

Northwestern University, Evanston, U.S.A.

K.A. Hahn, A. Kubik, N. Mucia, N. Odell, B. Pollack, A. Pozdnyakov, M. Schmitt, S. Stoynev, K. Sung, M. Trovato, M. Velasco, S. Won

University of Notre Dame, Notre Dame, U.S.A.

A. Brinkerhoff, N. Dev, M. Hildreth, C. Jessop, D.J. Karmgard, N. Kellams, K. Lannon, S. Lynch, N. Marinelli, F. Meng, C. Mueller, Y. Musienko ${ }^{33}$, T. Pearson, M. Planer, R. Ruchti, G. Smith, N. Valls, M. Wayne, M. Wolf, A. Woodard 
The Ohio State University, Columbus, U.S.A.

L. Antonelli, J. Brinson, B. Bylsma, L.S. Durkin, S. Flowers, A. Hart, C. Hill, R. Hughes, K. Kotov, T.Y. Ling, B. Liu, W. Luo, D. Puigh, M. Rodenburg, B.L. Winer, H.W. Wulsin Princeton University, Princeton, U.S.A.

O. Driga, P. Elmer, J. Hardenbrook, P. Hebda, S.A. Koay, P. Lujan, D. Marlow, T. Medvedeva, M. Mooney, J. Olsen, C. Palmer, P. Piroué, X. Quan, H. Saka, D. Stickland, C. Tully, J.S. Werner, A. Zuranski

Purdue University, West Lafayette, U.S.A.

V.E. Barnes, D. Benedetti, D. Bortoletto, L. Gutay, M.K. Jha, M. Jones, K. Jung, M. Kress, N. Leonardo, D.H. Miller, N. Neumeister, F. Primavera, B.C. Radburn-Smith, X. Shi, I. Shipsey, D. Silvers, J. Sun, A. Svyatkovskiy, F. Wang, W. Xie, L. Xu, J. Zablocki

Purdue University Calumet, Hammond, U.S.A.

N. Parashar, J. Stupak

Rice University, Houston, U.S.A.

A. Adair, B. Akgun, Z. Chen, K.M. Ecklund, F.J.M. Geurts, W. Li, B. Michlin, M. Northup, B.P. Padley, R. Redjimi, J. Roberts, J. Rorie, Z. Tu, J. Zabel

University of Rochester, Rochester, U.S.A.

B. Betchart, A. Bodek, P. de Barbaro, R. Demina, Y. Eshaq, T. Ferbel, M. Galanti, A. Garcia-Bellido, P. Goldenzweig, J. Han, A. Harel, O. Hindrichs, A. Khukhunaishvili, G. Petrillo, M. Verzetti, D. Vishnevskiy

The Rockefeller University, New York, U.S.A.

L. Demortier

Rutgers, The State University of New Jersey, Piscataway, U.S.A.

S. Arora, A. Barker, J.P. Chou, C. Contreras-Campana, E. Contreras-Campana, D. Duggan, D. Ferencek, Y. Gershtein, R. Gray, E. Halkiadakis, D. Hidas, E. Hughes, S. Kaplan, R. Kunnawalkam Elayavalli, A. Lath, S. Panwalkar, M. Park, S. Salur, S. Schnetzer, D. Sheffield, S. Somalwar, R. Stone, S. Thomas, P. Thomassen, M. Walker

University of Tennessee, Knoxville, U.S.A.

M. Foerster, K. Rose, S. Spanier, A. York

Texas A\&M University, College Station, U.S.A.

O. Bouhali ${ }^{63}$, A. Castaneda Hernandez, M. Dalchenko, M. De Mattia, A. Delgado, S. Dildick, R. Eusebi, W. Flanagan, J. Gilmore, T. Kamon ${ }^{64}$, V. Krutelyov, R. Montalvo, R. Mueller, I. Osipenkov, Y. Pakhotin, R. Patel, A. Perloff, J. Roe, A. Rose, A. Safonov, I. Suarez, A. Tatarinov, K.A. Ulmer

Texas Tech University, Lubbock, U.S.A.

N. Akchurin, C. Cowden, J. Damgov, C. Dragoiu, P.R. Dudero, J. Faulkner, S. Kunori, K. Lamichhane, S.W. Lee, T. Libeiro, S. Undleeb, I. Volobouev 


\section{Vanderbilt University, Nashville, U.S.A.}

E. Appelt, A.G. Delannoy, S. Greene, A. Gurrola, R. Janjam, W. Johns, C. Maguire, Y. Mao, A. Melo, P. Sheldon, B. Snook, S. Tuo, J. Velkovska, Q. Xu

\section{University of Virginia, Charlottesville, U.S.A.}

M.W. Arenton, S. Boutle, B. Cox, B. Francis, J. Goodell, R. Hirosky, A. Ledovskoy, H. Li, C. Lin, C. Neu, E. Wolfe, J. Wood, F. Xia

\section{Wayne State University, Detroit, U.S.A.}

C. Clarke, R. Harr, P.E. Karchin, C. Kottachchi Kankanamge Don, P. Lamichhane, J. Sturdy

\section{University of Wisconsin, Madison, U.S.A.}

D.A. Belknap, D. Carlsmith, M. Cepeda, A. Christian, S. Dasu, L. Dodd, S. Duric, E. Friis, B. Gomber, M. Grothe, R. Hall-Wilton, M. Herndon, A. Hervé, P. Klabbers, A. Lanaro, A. Levine, K. Long, R. Loveless, A. Mohapatra, I. Ojalvo, T. Perry, G.A. Pierro, G. Polese, I. Ross, T. Ruggles, T. Sarangi, A. Savin, N. Smith, W.H. Smith, D. Taylor, N. Woods

\section{$\dagger$ : Deceased}

1: Also at Vienna University of Technology, Vienna, Austria

2: Also at CERN, European Organization for Nuclear Research, Geneva, Switzerland

3: Also at Institut Pluridisciplinaire Hubert Curien, Université de Strasbourg, Université de Haute Alsace Mulhouse, CNRS/IN2P3, Strasbourg, France

4: Also at National Institute of Chemical Physics and Biophysics, Tallinn, Estonia

5: Also at Skobeltsyn Institute of Nuclear Physics, Lomonosov Moscow State University, Moscow, Russia

6: Also at Universidade Estadual de Campinas, Campinas, Brazil

7: Also at Centre National de la Recherche Scientifique (CNRS) - IN2P3, Paris, France

8: Also at Laboratoire Leprince-Ringuet, Ecole Polytechnique, IN2P3-CNRS, Palaiseau, France

9: Also at Université Libre de Bruxelles, Bruxelles, Belgium

10: Also at Joint Institute for Nuclear Research, Dubna, Russia

11: Also at Ain Shams University, Cairo, Egypt

12: Now at British University in Egypt, Cairo, Egypt

13: Now at Helwan University, Cairo, Egypt

14: Also at Suez University, Suez, Egypt

15: Also at Cairo University, Cairo, Egypt

16: Now at Fayoum University, El-Fayoum, Egypt

17: Also at Université de Haute Alsace, Mulhouse, France

18: Also at Brandenburg University of Technology, Cottbus, Germany

19: Also at Institute of Nuclear Research ATOMKI, Debrecen, Hungary

20: Also at Eötvös Loránd University, Budapest, Hungary

21: Also at University of Debrecen, Debrecen, Hungary

22: Also at Wigner Research Centre for Physics, Budapest, Hungary

23: Also at University of Visva-Bharati, Santiniketan, India

24: Now at King Abdulaziz University, Jeddah, Saudi Arabia

25: Also at University of Ruhuna, Matara, Sri Lanka

26: Also at Isfahan University of Technology, Isfahan, Iran 
27: Also at University of Tehran, Department of Engineering Science, Tehran, Iran

28: Also at Plasma Physics Research Center, Science and Research Branch, Islamic Azad University, Tehran, Iran

29: Also at Università degli Studi di Siena, Siena, Italy

30: Also at Purdue University, West Lafayette, U.S.A.

31: Also at International Islamic University of Malaysia, Kuala Lumpur, Malaysia

32: Also at CONSEJO NATIONAL DE CIENCIA Y TECNOLOGIA, MEXICO, Mexico

33: Also at Institute for Nuclear Research, Moscow, Russia

34: Also at Institute of High Energy Physics and Informatization, Tbilisi State University, Tbilisi, Georgia

35: Also at St. Petersburg State Polytechnical University, St. Petersburg, Russia

36: Also at National Research Nuclear University 'Moscow Engineering Physics Institute' (MEPhI), Moscow, Russia

37: Also at California Institute of Technology, Pasadena, U.S.A.

38: Also at Faculty of Physics, University of Belgrade, Belgrade, Serbia

39: Also at Facoltà Ingegneria, Università di Roma, Roma, Italy

40: Also at Scuola Normale e Sezione dell'INFN, Pisa, Italy

41: Also at University of Athens, Athens, Greece

42: Also at Warsaw University of Technology, Institute of Electronic Systems, Warsaw, Poland

43: Also at Institute for Theoretical and Experimental Physics, Moscow, Russia

44: Also at Albert Einstein Center for Fundamental Physics, Bern, Switzerland

45: Also at Gaziosmanpasa University, Tokat, Turkey

46: Also at Mersin University, Mersin, Turkey

47: Also at Cag University, Mersin, Turkey

48: Also at Piri Reis University, Istanbul, Turkey

49: Also at Adiyaman University, Adiyaman, Turkey

50: Also at Ozyegin University, Istanbul, Turkey

51: Also at Izmir Institute of Technology, Izmir, Turkey

52: Also at Mimar Sinan University, Istanbul, Istanbul, Turkey

53: Also at Marmara University, Istanbul, Turkey

54: Also at Kafkas University, Kars, Turkey

55: Also at Yildiz Technical University, Istanbul, Turkey

56: Also at Kahramanmaras Sütcü Imam University, Kahramanmaras, Turkey

57: Also at Rutherford Appleton Laboratory, Didcot, United Kingdom

58: Also at School of Physics and Astronomy, University of Southampton, Southampton, United Kingdom

59: Also at Utah Valley University, Orem, U.S.A.

60: Also at University of Belgrade, Faculty of Physics and Vinca Institute of Nuclear Sciences, Belgrade, Serbia

61: Also at Argonne National Laboratory, Argonne, U.S.A.

62: Also at Erzincan University, Erzincan, Turkey

63: Also at Texas A\&M University at Qatar, Doha, Qatar

64: Also at Kyungpook National University, Daegu, Korea 